\title{
Bendy: An Exploration into Gaming with Mobile Flexible Devices
}

\author{
by
}

\author{
Jessica Lo
}

A thesis submitted to the Faculty of Graduate and Postdoctoral Affairs in partial fulfillment of the requirements for the degree of

\author{
Masters of Applied Sciences \\ in \\ Human Computer Interaction
}

Carleton University

Ottawa, Ontario

(C) 2013, Jessica Lo 


\begin{abstract}
We explore gaming with mobile flexible devices through a comprehensive study. We conducted two experiments with two commonly sized mobile devices. The first experiment created user-defined bend gestures from a set of tasks derived from gaming. The results suggest pairing opposing events by gesture location, little differences in gestures performed on two sized devices and interaction paradigms relating to the Simon Effect. The results of the first experiment were summarized and provided guidelines for the design of the game controls used in the second experiment. We implemented the userdefined gestures into 6 games using an interactive flexible prototype. Usability and user experience were evaluated and we find an overall preference for the small size, identified an important usability issue related to hand position and interaction paradigms regarding the Simon Effect and bimanual input. Finally, we propose a set of design recommendations for flexible device interactions and game designers.
\end{abstract}




\section{Acknowledgements}

This thesis would not have been possible without the brilliant and wonderful people who have gone above and beyond. My accomplishments are solely attributed to all the exceptional people in my life. In particular, I thank Alex Eady for his refreshing optimism, comedic company and nimble fingers during the prototyping process. Thank you to Daniel McCarney for his superior coding abilities and bringing Bendy to fruition. I thank Whitney Payne for her encouragement and support through the writing blues. And last but not least, I thank Andy Sharpe for taking care of me through broken bones, feeding me through hungry late nights, enduring the wrath of this thesis and always and forever keeping me in good spirits. Moreover, I thank Penelope Chow, Michelle Amore and Shayta Roy whom without fail continue support me through any endeavor and encourage me through all the goals I set forth.

Above all, I thank my advisor, Audrey Girouard. Without her tremendous guidance, unequivocal support and enduring patience, I would not have had the spirit and ambition to complete this research. I thank her on both the academic and personal level for the growth I have achieved through this thesis. I am extremely grateful to have had an advisor whom accepts and embraces my creative ways and has enabled achievements beyond my own expectations. She has thrown me over hurdles, put out fires and provided solace through all the challenges. I appreciate her ability to understand my thoughts through my inability to express them explicitly. And most importantly, I thank her for all the smiles, good laughs and memories we shared together over the past year. 


\section{Table of Contents}

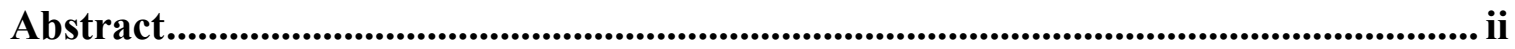

Acknowledgements ............................................................................................ iii

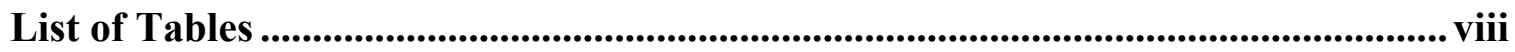

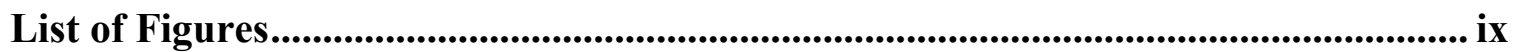

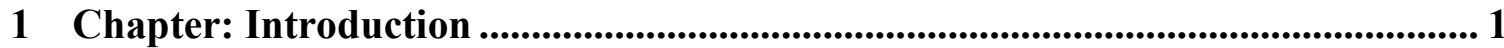

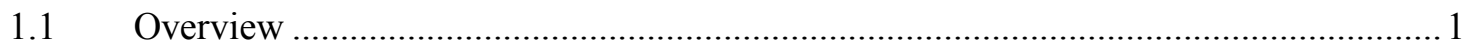

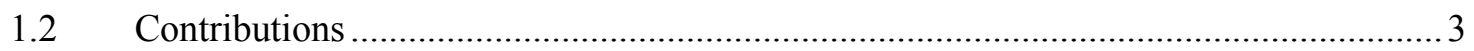

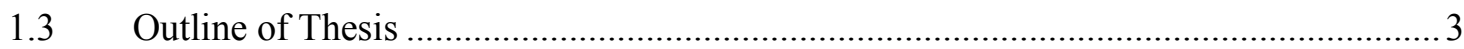

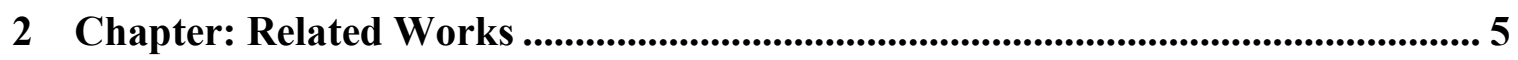

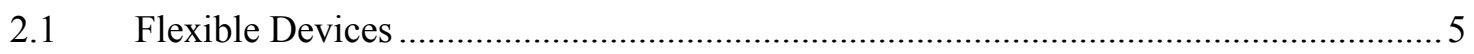

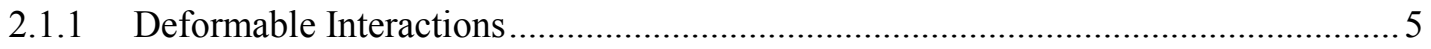

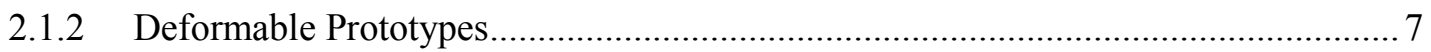

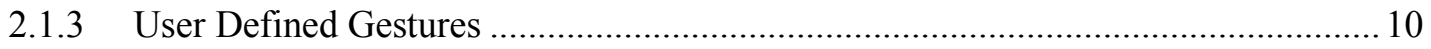

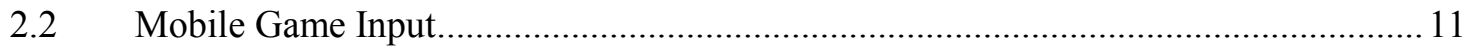

2.3 Stimulus-Response Compatibility: The Simon Effect............................................. 13

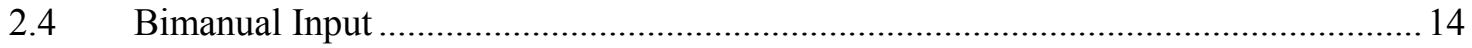

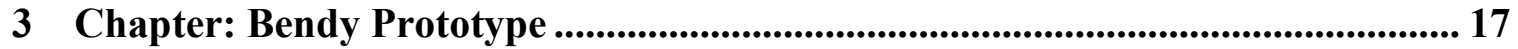

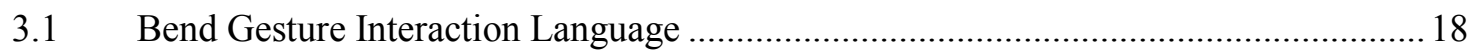

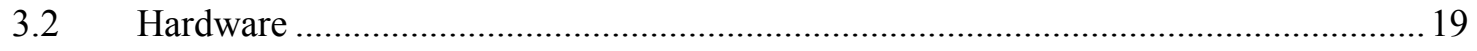

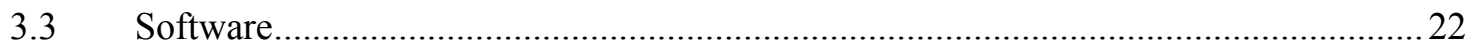

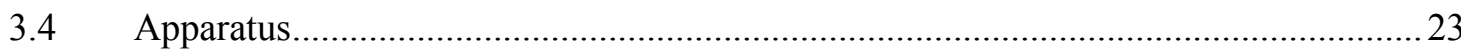

4 Chapter: Experiment 1: User-Defined Bend Gestures for Gaming ...................... 24

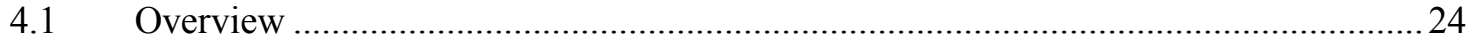

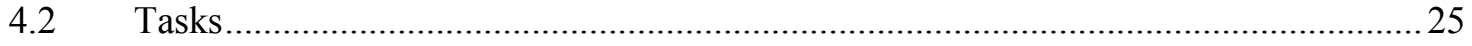




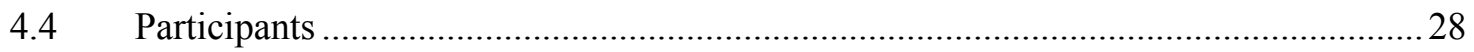

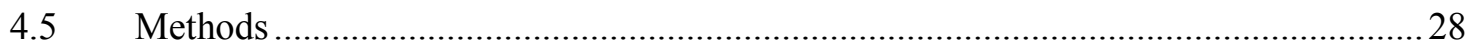

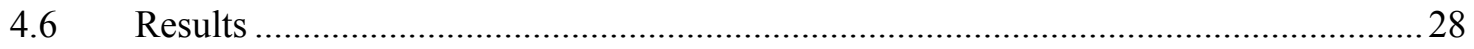

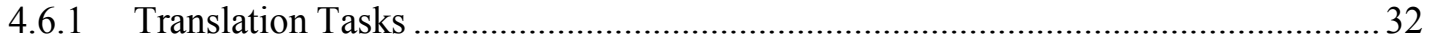

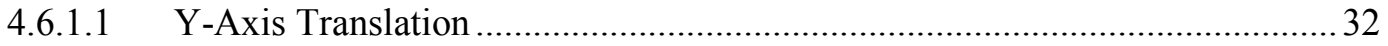

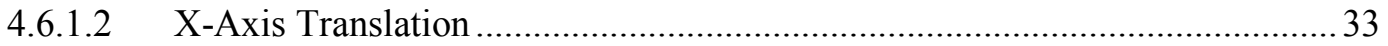

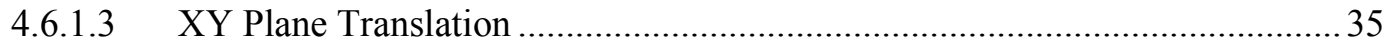

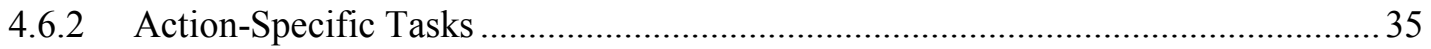

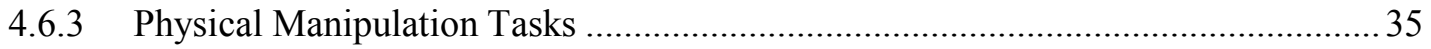

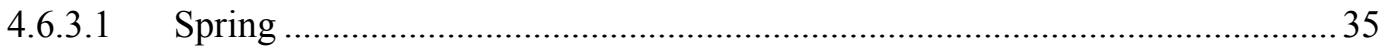

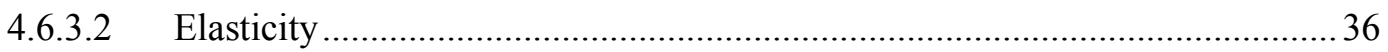

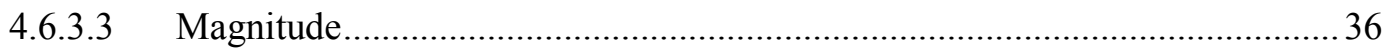

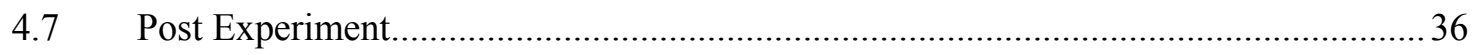

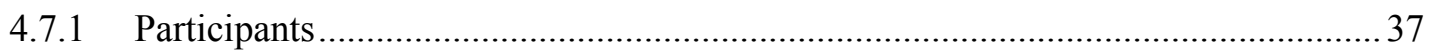

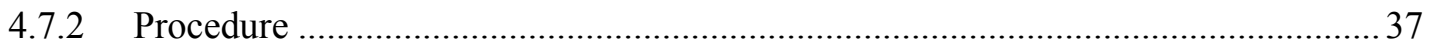

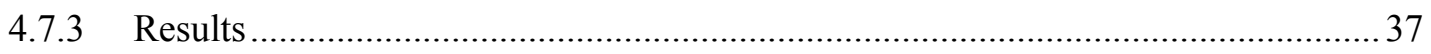

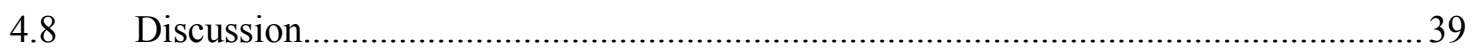

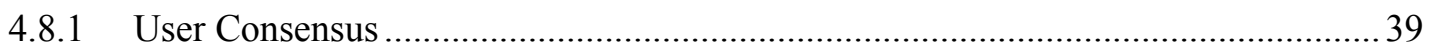

4.8.2 Opposing Events with Paired Gestures .............................................................. 40

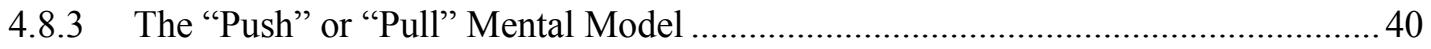

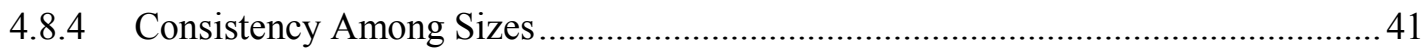

4.8.5 Simon Effect: Collocation of Stimulus and Gesture ............................................. 42

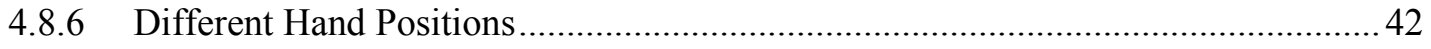

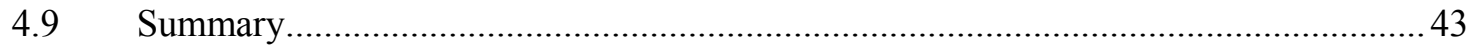

5 Chapter: Experiment 2: Evaluating Usability and User Experience.................... 45

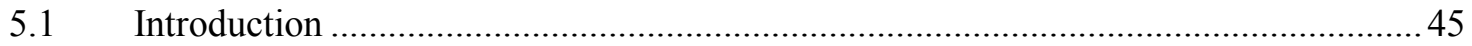




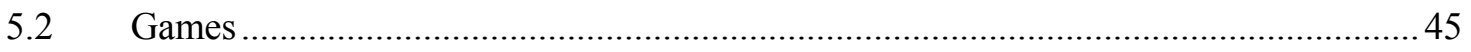

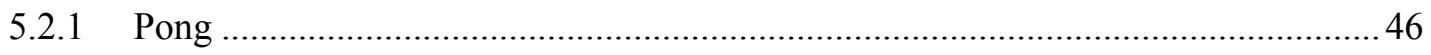

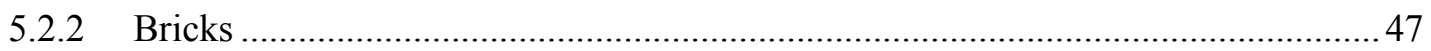

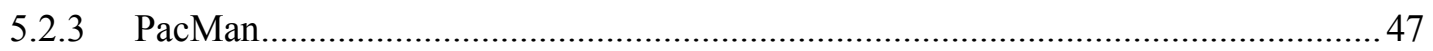

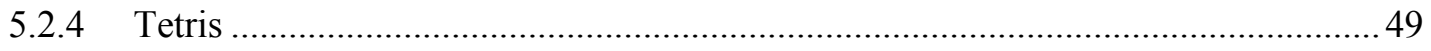

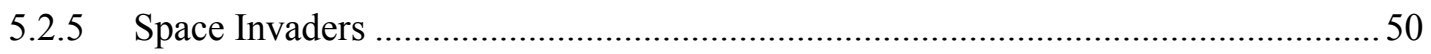

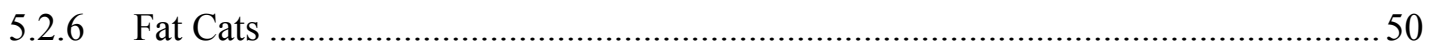

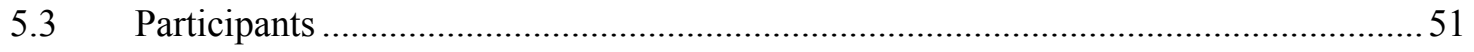

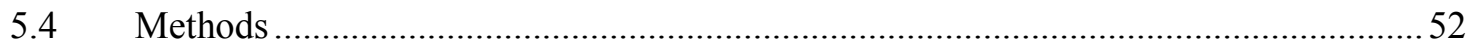

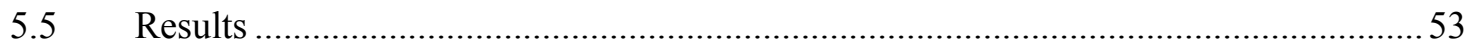

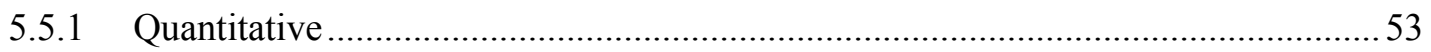

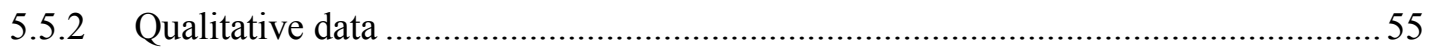

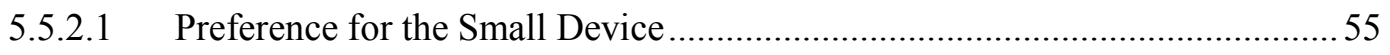

5.5.2.2 Reducing the Need to Reposition Hands to Perform Gestures.........................56

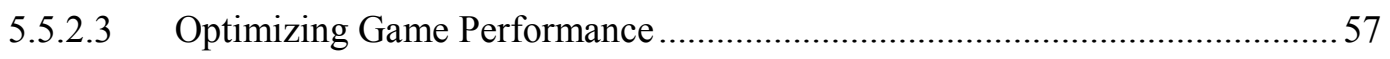

5.5.2.4 Observable Patterns of the Simon Effect......................................................... 58

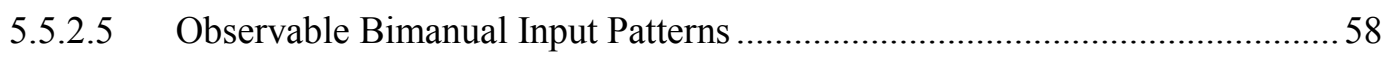

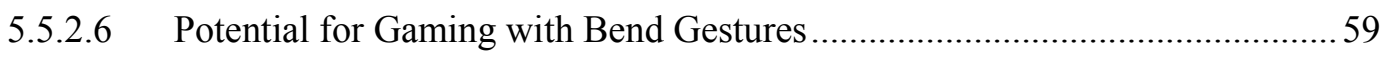

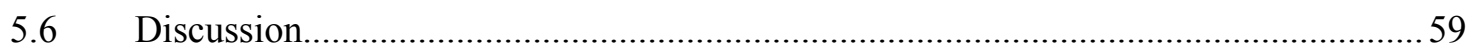

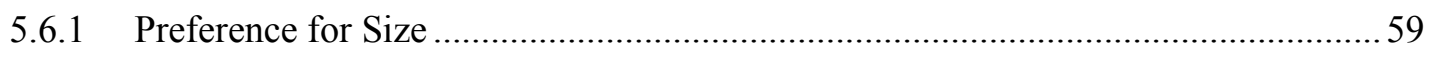

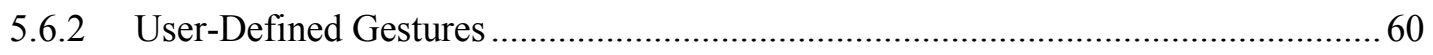

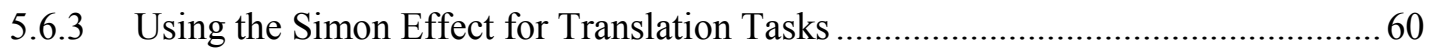

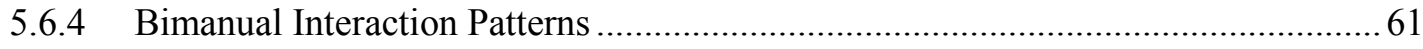

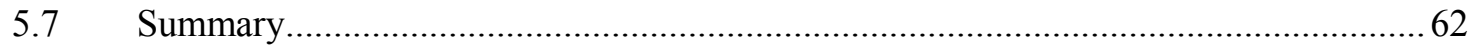

6 Chapter: Conclusion ............................................................................................ 63

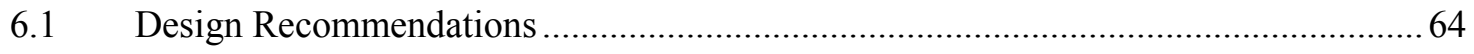




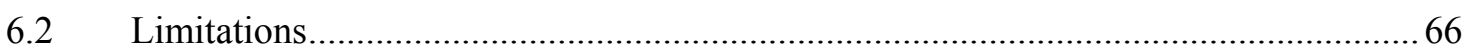

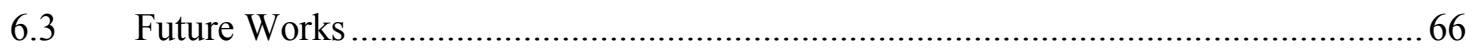

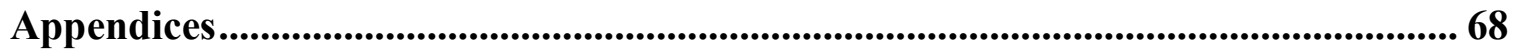

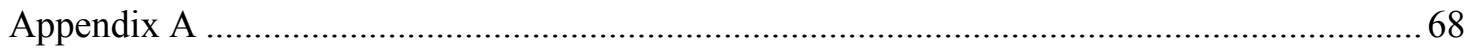

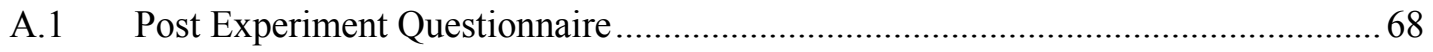

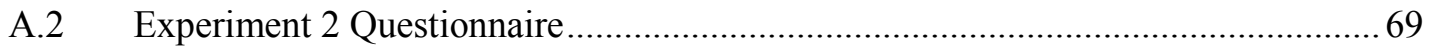




\section{List of Tables}

Table 1 Gestures performed for $y$-axis translation for both sizes. .............................. 33

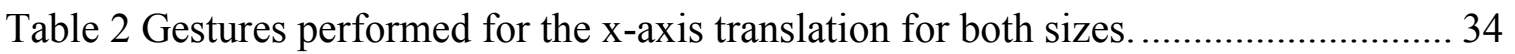

Table 3 Unresolved gesture conflicts in the first experiment. ................................... 36 


\section{List of Figures}

Figure 1 Gaming with mobile flexible devices......................................................... 2

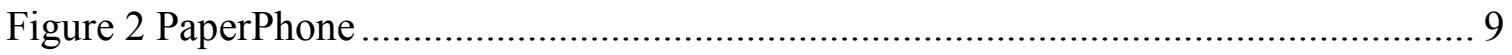

Figure 3 An example of spatial correspondence........................................................ 13

Figure 4 Illustration of the Simon Task ...................................................................... 14

Figure 5 Super Nintendo game console controller. .................................................. 16

Figure 6 Small and large Bendy prototypes compared to mobile devices....................... 17

Figure 7 Bendy's bend gesture interaction language................................................... 18

Figure 8 Exploded view of the Bendy prototype..................................................... 19

Figure 9 Iterative prototypes using different material stiffness and compositions ........... 20

Figure 10 Flexible printed circuit ………………………......................................... 21

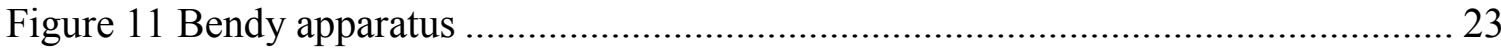

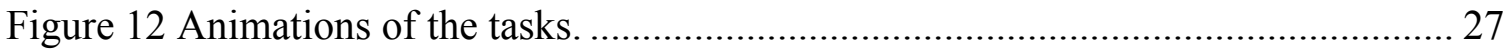

Figure 13 Agreement scores for both device sizes..................................................... 30

Figure 14 Frequency plot of the gestures collected. ....................................................... 31

Figure 15 User-defined gesture sets including unresolved conflicts. .............................. 38

Figure 16 The "push" or "push" mental models............................................................... 41

Figure 17 Illustration of how some users held the prototypes....................................... 43

Figure 18 Game controls for Pong............................................................................ 46

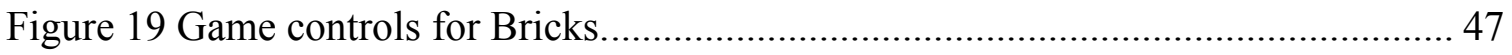

Figure 20 Game controls for PacMan....................................................................... 48

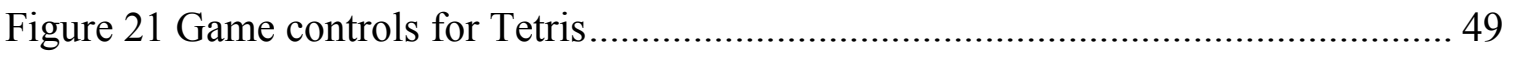

Figure 22 Game controls for Space Invaders.......................................................... 50 
Figure 23 Game controls for Fat Cats................................................................... 51

Figure 24 Averages of physical ease of use, mental intuitiveness and fun for all games. 55

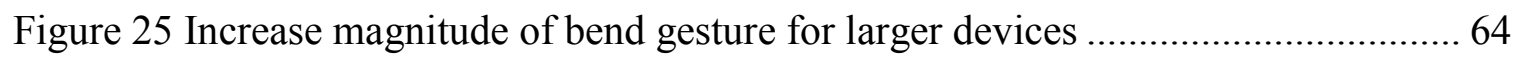




\section{List of Appendices}

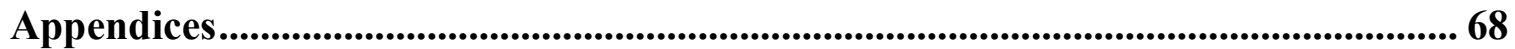

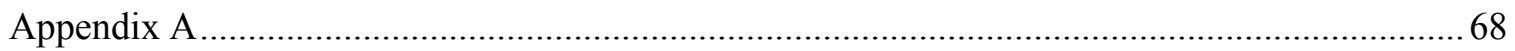

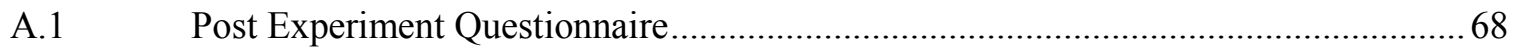

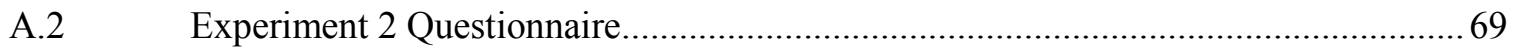




\section{Chapter: Introduction}

\subsection{Overview}

New technologies that allow different and unexplored input modalities present an opportunity to create novel interactions [34]. With the advent of flexible devices, there is an abundance of interaction opportunities surrounding deformation. We use deformation to interact with everyday objects which allows for a rich set of possibilities, involving many degrees of freedom with intuitive interactions [37]. Flexible devices benefit from these attributes by connecting reality based interactions with virtual experiences, which embody engaging interactions $[9,17,19,20]$. While several studies have explored deformation within the context of mobile applications, few researchers have investigated mobile games.

Mobile games are designed to be short, simple and competitively engaging, which makes them the most popular mobile applications [41]. The mobile device as a gaming platform has gained more users and is growing more rapidly than any other platform [28]. These games appeal to almost every demographic, are easy to play, offer instant gratification and require minimal commitment in both time and cost [6]. In addition, the proliferation of mobile device ownership has made mobile games more accessible. The smaller display size of mobile devices restricts the use of on screen game controls and limits game interaction. Moreover, an inherent limitation of touch input is finger occlusion, commonly known as the "fat fingers" problem [35]. By leveraging the use of deformation as an input method and the characteristics of mobiles games, we envision an input modality that could potentially create new and innovative gameplay while resolving screen occlusion issues (Figure 1). 
As flexible devices have a great potential for gaming, we present Bendy, an explorative study into the interactions of gaming with mobile flexible devices using a flexible interactive prototype. Amongst the rise of smartphone ownership there has also been a growth in tablet and eReader consumption. Our main research goal is to explore, discover and illuminate the intricate interactions in gaming with bend gestures. We examine the differences in two commonly sized mobile devices. To explore user behaviour, we conducted a participatory experiment where users were presented with a series of 2D visual stimuli derived from simple games and asked to define bend gestures for each. We quantified the results and created user-defined bend gesture sets then implemented them into six games. Deformation is a rich interaction language with inherent complex user interactions. We built the Bendy prototype to provide users with an immersive interactive experience and gathered feedback regarding user experience and the usability of bend gestures. The second experiment examined the detailed user interactions during gaming and revealed novel interaction paradigms. We conclude by reporting insights found and propose design recommendations for designing games with mobile flexible devices.

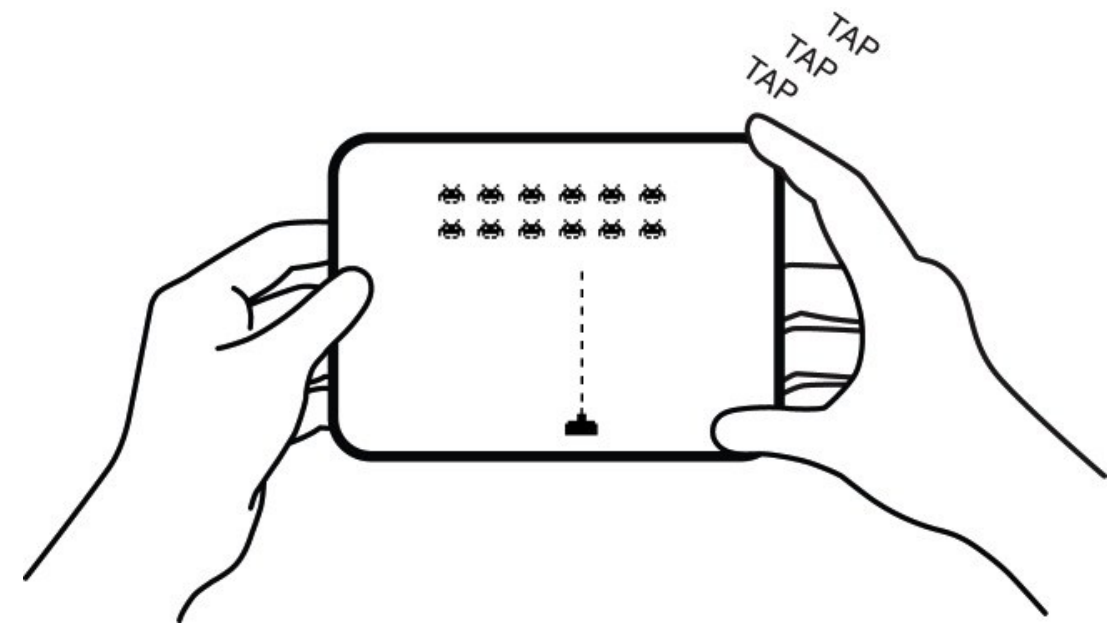

Figure 1 Gaming with mobile flexible devices. 


\subsection{Contributions}

We contribute to deformable interaction research by presenting Bendy, a fully flexible device that differs from prior form factors that have been studied, in that its flexibility affords it a greater degree of freedom and therefore allows a richer interaction language. The Bendy prototype was designed through an iterative process that explored different materials and physical affordances to emulate a real product. As a result, Bendy is a robust flexible prototype that allows for in-depth deformable interaction research in the absence of true flexible display technology. The two experiments revealed new interaction paradigms that have not been discussed with regards to flexible devices in previous research. Additionally, there was an extremely positive response from users regarding the use of bend gestures as an input method for gaming. This confirmed the potential use of bend gestures for gaming and builds a case for further research. In addition, this study examines the differences between two common sizes of mobile device, which has not been studied before. We put forth several design recommendations for flexible devices and game designers.

\subsection{Outline of Thesis}

This thesis is divided into six chapters. The first chapter introduces the context of our study and intentions for exploring gaming with mobile flexible devices. The following chapter reviews prior works related to our research in the areas of flexible devices, mobile games, stimulus-response compatibility and bimanual input.

The third chapter presents our apparatus and the methods used to create a flexible and robust prototype. This chapter provides prototype design details, assembling the hardware components, and writing the software used to detect bend gestures. 
The fourth chapter provides detail about our first experiment, where participants were asked to create bend gestures given a $2 \mathrm{D}$ visual stimulus. This chapter includes descriptions of the tasks, participants, procedure, results and discussion.

The fifth chapter details our second experiment, where participants played and evaluated six games using the user-defined gestures created in the first experiment. This chapter includes descriptions of the tasks, participants, procedure, results and discussion.

The final chapter summarizes our findings and proposes design recommendations for flexible devices and game designers. We discuss the limitations of our research and provide suggestions for future works. 


\section{Chapter: Related Works}

With technological advances in flexible displays, there has been increasing interest in studying deformable interactions. We review related works on interactions with flexible devices, input modalities in mobile games, user-defined gesture studies, stimulus-response compatibility and bimanual input.

\subsection{Flexible Devices}

Deformable user interfaces use the physical deformation of an object as a form of input [23]. There are many intricate aspects to studying flexible devices, from the physical properties of the device itself to the information processes users experience during interactions. We surveyed prior works that have explored many of these intricacies using different methodologies and prototypes and summarize their findings.

\subsubsection{Deformable Interactions}

Deformable user interfaces allow many different interactions from twisting to scrunching. In addition to diverse interactions techniques, researchers have also explored several different applications of flexible displays.

ShapeTape [4] was one of the earliest works that explored deformation as a user interface. Users could perform continuous bends and twists using an elongated rubber tape as an external input device to manipulate 3D computer models. ShapeTape allowed users to directly manipulate virtual surfaces using a physical object. Following ShapeTape was another influential work by Schwesig et al [34], who envisioned a bendable computer, Gummi; a concept which was based on the physical deformation of a handheld device. The Gummi user interface formed a basic interaction language using simple bend gestures and a 2D position controller. The study implemented the interaction 
techniques with several different tasks such as map navigation, menu navigation, media player control and text input. The authors also explored using deformation in a simple game where it was used to control the game character's movement. Schwesig et al. observed that users could easily grasp the basic interaction principles and had no difficulty in using it with certain applications. This pioneering research proves that deformable interaction is a viable concept.

The next body of works examined flexible devices using paper metaphors. Paper metaphors have been extensively used because of the similar physical affordances they share with flexible devices, which creates an intuitive interaction experience. For instance, Bookisheet [43] examined user behaviour when navigating a book and quantified the interactions using the forces applied by the thumb and the elastic energy of the bent surface. As a result, Bookisheet translated the basic operations of navigating through a book and designed an interface based on these metaphors for a dual and single display prototype. The authors concluded that users achieved the same level of performance as seen in conventional devices as they did with their flexible prototype. Tajika et al. [39] conducted a similar study but focused mainly on building an algorithm for implementation, which was based on direction of operation, bend thresholds and time. Bendflip [44] examined the influences of physical form factors and the various modes of input for flexible eReaders. They compared touch, pressure, and bend input between rigid and flexible devices. Their results suggest that bend input is comparable to button input for navigation on flexible devices. Following these works, researchers broadened their interests to other applications such as smartphones [24], television remotes [26], gaming [47] and media players [22]. 
Cobra [47] introduced flexible displays in a gaming context through the use of bend gesture input. They proposed a portable system comprised of a shoulder-mounted pico-projector connected to a laptop, which recognized user input from the flexible device. Their preliminary exploration did not expand on game interactions and omitted a user evaluation. Our work hopes to extend beyond the concept proposed by Cobra and evaluate gaming with flexible devices in detail.

Other works have explored different deformation techniques other than bending with non-specific applications. For example, Gallant et al. [12] conducted a study using paper prototypes and explored other techniques such as bending, folding, scooping, hovering, leafing, shaking and squeezing. Lee et al. [27] conducted a study where users performed deformations on plastic, elastic cloth and paper. In addition to bending, users created gestures using twisting, folding, stretching and scrunching. These studies demonstrate deformation is a rich interaction language beyond simple bends.

This body of research illustrates the diversity of deformation as an interaction language. It can consist of a few simple bend gestures to more complex interactions. For our study, we drew upon prior works to build the interaction language for the Bendy prototype.

\subsubsection{Deformable Prototypes}

Research with flexible devices can be limiting, as it is often difficult to implement the technology. Despite this limitation, researchers have approached studying deformable interactions using several techniques for visual feedback and deformation sensing.

Twend [15] embedded optical sensors in between two $130 \mathrm{~mm} \times 50 \mathrm{~mm}$ pieces of flexible plastic. Similarly, ShapeTape [4] also used optical sensors but comprised of a 
spring steel core and measured $960 \mathrm{~mm} \times 10 \mathrm{~mm}$. Both prototypes were connected to an external display and were used as an external input device.

In the absence of flexible displays, several prototypes affixed an external rigid display onto a piece of flexible plastic augmented with bend sensors, which enable deformable interactions and eliminate the gulf of execution. Gummi [34] built a handheld sized prototype using a small TFT display which was mounted on top of a piece of flexible plastic augmented with bend sensors and a 2D position controller. Tajika et al. [39] built a similar prototype, but used a larger piece of plastic with bend sensors, which enclosed a small LCD monitor. BendFlip [44] used a $203.2 \mathrm{~mm}$ x $177.8 \mathrm{~mm}$ flexible plastic, which was built with bend, touch and pressure sensors that were mounted behind a 6" LCD screen.

Another system commonly used is the combination of camera sensing and projection. Gallant et al. [12] devised a rapid prototyping method by using black cardstock with infrared reflective markers. To detect the markers, they modified a webcam with infrared LEDs around the lens. This prototype was used as an external input device. Watanabe et al. [43] also explored the use of infrared reflective markers and cameras, however the markers were used to detect the position and orientation of their prototype in order to project images onto the display. Cobra [47], a mobile gaming system, used a shoulder mounted pico-projector and the prototype was tracked using infrared LEDs detected by a Wii Remote. FlexPad [37] provided high fidelity deformation sensing by using a ceiling mounted depth sensing camera and projector. We utilize similar projection methods to provide our users with visual feedback. 
PaperPhone [24] and Kinetic from Nokia Research [22] use active flexible displays in their handheld sized prototypes. PaperPhone was built using a 3.7" electrophoretic display augmented with bend sensors. The Kinetic prototype consists of a flexible OLED display with two rigid parts affixed to the sides and uses a strain gauge to detect deformation.

In early 2013, Samsung officially launched their YOUM flexible OLED (organic light emitting diode) display technology [33]. They demonstrated with a working prototype as well as a rigid mobile device with a curved display. While flexible displays are not commercialized yet, flexible display technology is advancing rapidly.

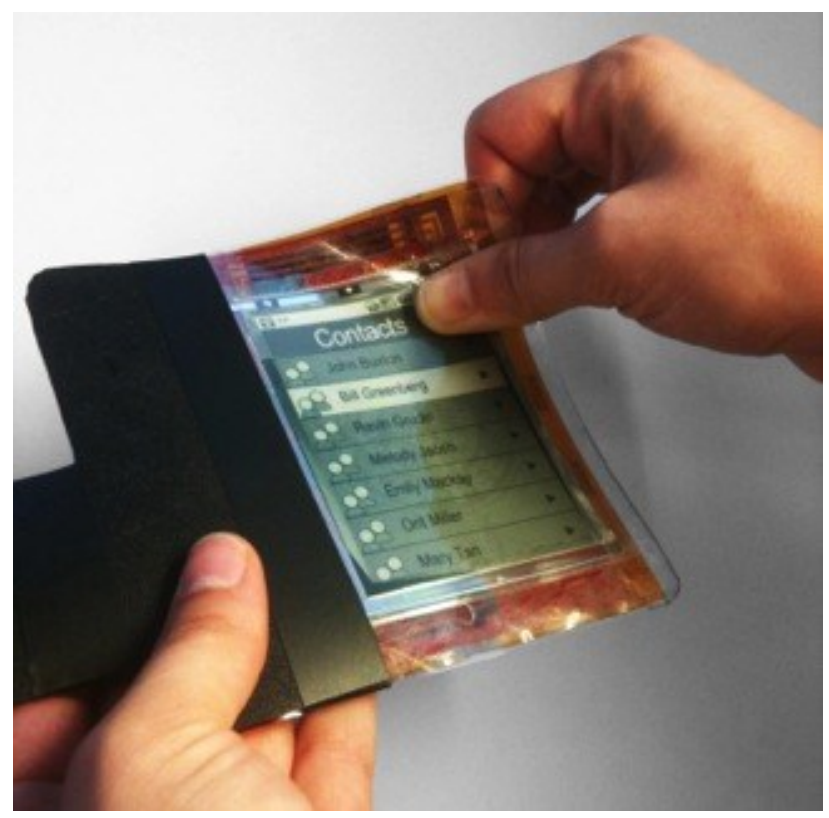

Figure 2 PaperPhone [24]

There are three common sizes of flexible prototypes that have been studied: small (handheld), medium (eReader), and large (A4 paper or tablet). Lee et al. [25] conducted a study to examine the effects between small $(59 \mathrm{~mm} \times 115 \mathrm{~mm})$ and large $(210 \mathrm{~mm} \mathrm{x}$ $297 \mathrm{~mm}$ ) sized prototypes. They found a user preference for the small sized device and that users operated most commonly in landscape orientation. 
Prototypes can also vary significantly in their material composition and construction. Kildal et al. [23] conducted a study to examine the effects of material stiffness using the Kinetic prototype. While they did not find that the range of material stiffness influenced user performance, there was a strong user preference for less stiff materials. Users also felt more comfortable and engaged with softer materials.

We leveraged a combination of techniques and findings from this body of research in the design and development of the Bendy prototype and apparatus. The Bendy prototype was fabricated with less stiff material and purposed to be used in the landscape orientation. We used projection and marker tracking for visual display.

\subsubsection{User Defined Gestures}

User-defined gestures outperform those created by designers as shown by Wobbrock et al. [45], who proposed a guessability study methodology in creating userdefined gestures for touch input. The idea underlying the methodology is to observe user behaviour given the effects, the visual feedback of the system, and ask users to create the cause, the gesture itself. The process creates a dialogue between the system and users' behaviour enabling users to provide accurate response selections and provides insights into user patterns and mental models. The gestures are then grouped and an agreement score is calculated which is represented in a single numeric value, which represents the degree of consensus amongst participants for each task. As a result, the gesture with the highest agreement score is chosen for the task.

PaperPhone [24] applied this methodology in their study and asked participants to create and evaluate bend gestures for smartphone related tasks such as navigating through a contact list, calling a contact and media player navigation. The study was divided into 
three sessions. The first session asked participants to create eight unique bend gesture pairs. Participants were then asked to test the bend gestures with a set of paired tasks. In the third session, the researchers applied the bend gesture pairs to an application where the tasks were cohesive. The authors provide detailed criterion for building a bend gesture interaction language using consistency, orthogonality, polymorphism, redundancy and directionality.

Lee et al. [27] also applied the guessability methodology to their study. They explored deformation gestures beyond simple bends and twists by presenting users with A4 sized plastic, elastic cloth and paper mock prototypes. They categorized seven types of deformation: bending, twisting, folding, rolling, crumpling, tearing, and stretching. Participants were asked to create deformation gestures using the three materials with eleven commands. The authors found users used common daily interactions with real objects to define their gestures and that user preference and agreement increased for materials with more flexibility. We apply this methodology to our study by leveraging Lee's finding, as it is important to allow users to define potential gestures for flexible displays.

\subsection{Mobile Game Input}

The most common form of game input on current mobile devices is touch, which, in conjunction with small display size, often limits game interaction since small display sizes restrict the use of on screen game controls. Additionally, the most common problem with touch interfaces is the "fat fingers" problem [35], which is the effect of screen occlusion caused by finger position.

Zaman et al. [48] compared the use of touchscreen-based controls on the Apple 
iPhone versus physical controls on the Nintendo DS for the game Assassin's Creed. They found users' performance and preference were significantly higher when using physical buttons. This suggests that designers need to consider alternative input methods for touchscreen devices.

Several comparative studies have explored the use of other input modalities versus touch input. The accelerometer, found in current mobile devices, has been used as an alternative input modality for gaming. Chehimi et al. [7] explored the use of accelerometers in a 3D space shooter game and found the response from users to be positive and intuitive. Gilbertson et al. [13] compared the use of an accelerometer versus a physical joystick button in a 3D first person driving game and found an extremely positive response towards accelerometers as being more enjoyable. Browne et al. [5] evaluated usability and user experience of a side-scrolling shooter game for the iPod Touch using three inputs: accelerometer, simulated touch button and touch gestures. Users preferred the accelerometer-based interface and it also exhibited the best performance measures. Browne et al. recommend that the physical properties of gestures should be directly translated into the game's virtual properties. While using the accelerometer has been widely accepted, tilting or shaking the mobile device can obstruct the field of view. Böhrs et al. [8] proposed a comparative study to investigate the effectiveness of a button-less interface using the combination of touch and accelerometer input. They designed a touch interface with accelerometer input, touch interface without accelerometer input and on-screen touch button interface to use with a labyrinth game. This work illustrates the necessity of exploring other input modalities, which offer ways of improving touch input's shortcomings. 


\subsection{Stimulus-Response Compatibility: The Simon Effect}

In order to fully understand the correlation of bimanual input and visual stimulus we consider the Simon Effect [36]. Stimulus-response compatibility [31] is a psychological phenomenon where the rate of information transfer is dependent on the association of stimulus and response. It is often measured in response times, frequency of errors or learnability. In human computer interaction, the more commonly known stimulus-response compatibility theories are Fitt's Law [10] and the Hick-Hyman Law $[16,18]$.

The relationship of stimulus and response can be either compatible or incompatible. An example of spatial correspondence is the task of picking up a coffee mug with your right hand (Figure 3).

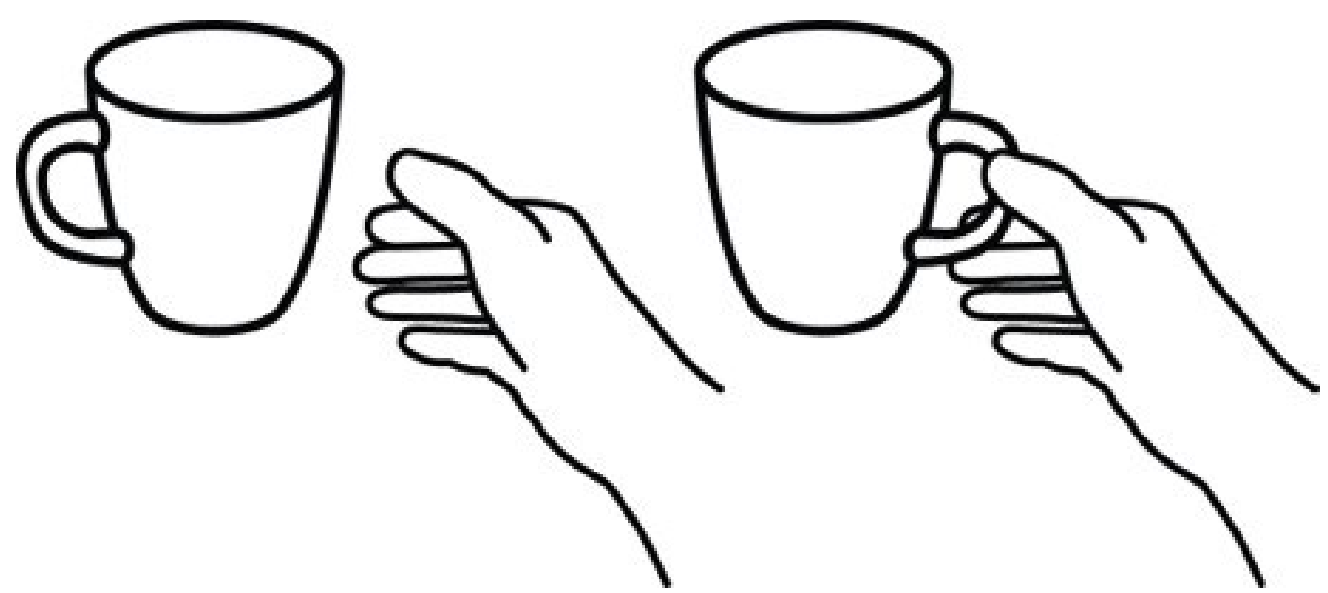

Figure 3 An example of spatial correspondence.

If the handle of the coffee mug were oriented on the left side, you would need to re-orient the handle towards your right hand. This is an example of spatial incompatibility. The handle (stimulus) of the coffee mug is incompatible with your right hand. This stimulus-response compatibility in spatial correspondence is known as the 
Simon Effect (Figure 4) [36]. Simon first discovered this effect when he presented participants with auditory words "left" and "right" through the left and right speakers of a set of headphones. Participants were to respond to the meaning of the word by pressing left and right keys, but needed to ignore the location of the sound. Participants responded faster when the location of the word corresponded spatially to the response. The Simon Effect is relevant in this work as it may influence the location of the gesture in relation to the location of the visual stimuli.
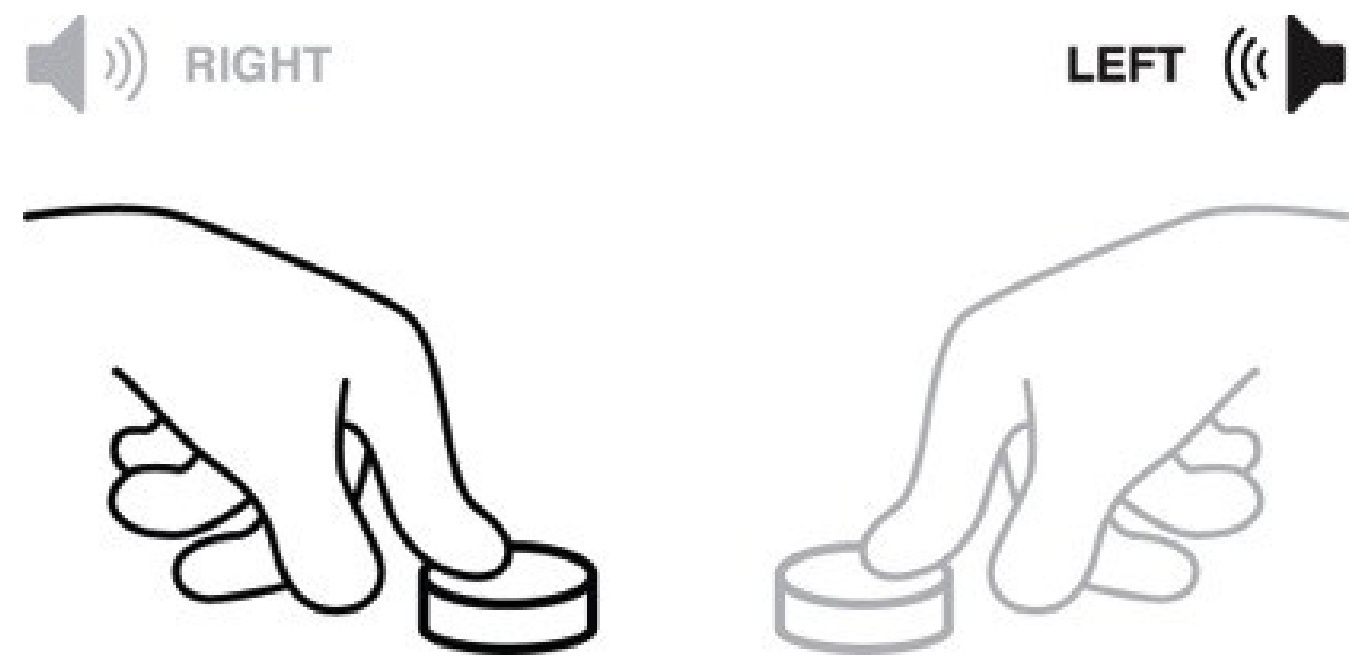

Figure 4 Illustration of the Simon Task

\subsection{Bimanual Input}

The fully flexible form factor of Bendy utilizes bimanual input and, therefore, we utilize Guiard's Kinematic Chain Model [14], a set of principles for two-handed activities. Bimanual interactions are categorized as symmetrical or asymmetrical tasks. Examples of symmetrical tasks are jumping rope or weight lifting, whereas an asymmetrical task is handwriting. 
The general principles of the Kinematic Chain Model are:

1. Motion of the preferred hand finds spatial references to the non-preferred hand. In the example of handwriting, the non-preferred hand controls the position of the paper and the preferred hand acts in reference to the position of the non-preferred hand.

2. The preferred and non-preferred hands contrast in the scale of motion. This principle states the preferred hand is used for more frequent and detailed actions, whereas the non-preferred hand performs coarse actions. In the example of handwriting, the non-preferred hand orients the paper, the coarse action, and the preferred hand performs the more "detailed" task of handwriting.

3. The non-preferred hand begins its action before the preferred. Again, using the example of handwriting, the non-preferred hand positions the paper before the preferred hand begins writing.

In the context of gaming, Guiard's principles are applied to the design of game controllers. For the past two decades, game controllers have consistently been designed the same way with the directional pad on the right and the action buttons on the left (Figure 5). Some have suggested the design is justified using Guiard's principles, where "coarse" actions, such as manipulating direction, are mapped to the non-preferred hand and more complex actions are produced by the preferred hand. 


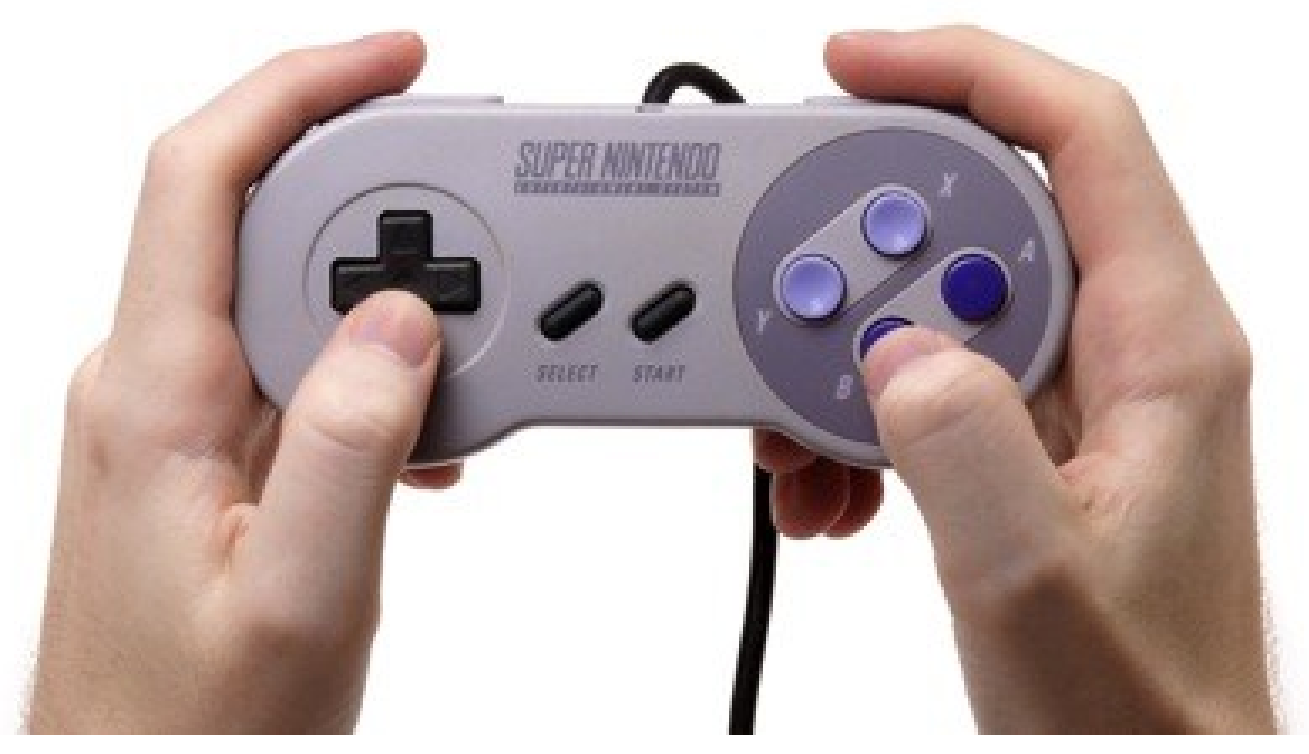

Figure 5 Super Nintendo game console controller. [49]

The Bendy prototype's form factor requires users to perform bimanual activities during game play, We explored different game commands in our study, which involved both directionality and actions, this body of research helped inform us of user behaviour and patterns. 


\section{Chapter: Bendy Prototype}

While flexible devices do not currently exist in the consumer market, we felt it was valuable to create a prototype that would emulate a real product. Furthermore, it was imperative that we provide users with an interactive gaming experience. The Kinetic prototype [22] served as a guideline for our design. The Bendy prototype contains no rigid parts, which enables an increase in the number of degrees of freedom and bend gesture interaction language available in comparison to the other flexible prototypes. We fabricated the flexible prototypes in two sizes, small $(120 \mathrm{~mm} \times 72 \mathrm{~mm})$ and large $(170 \mathrm{~mm}$ x 120mm), using Flexpoint [11] bidirectional bend sensors (Figure 6). The composition of Bendy consists of a thin sheet of polycarbonate plastic with the flexible circuit enclosed in a thin layer of silicon resin.
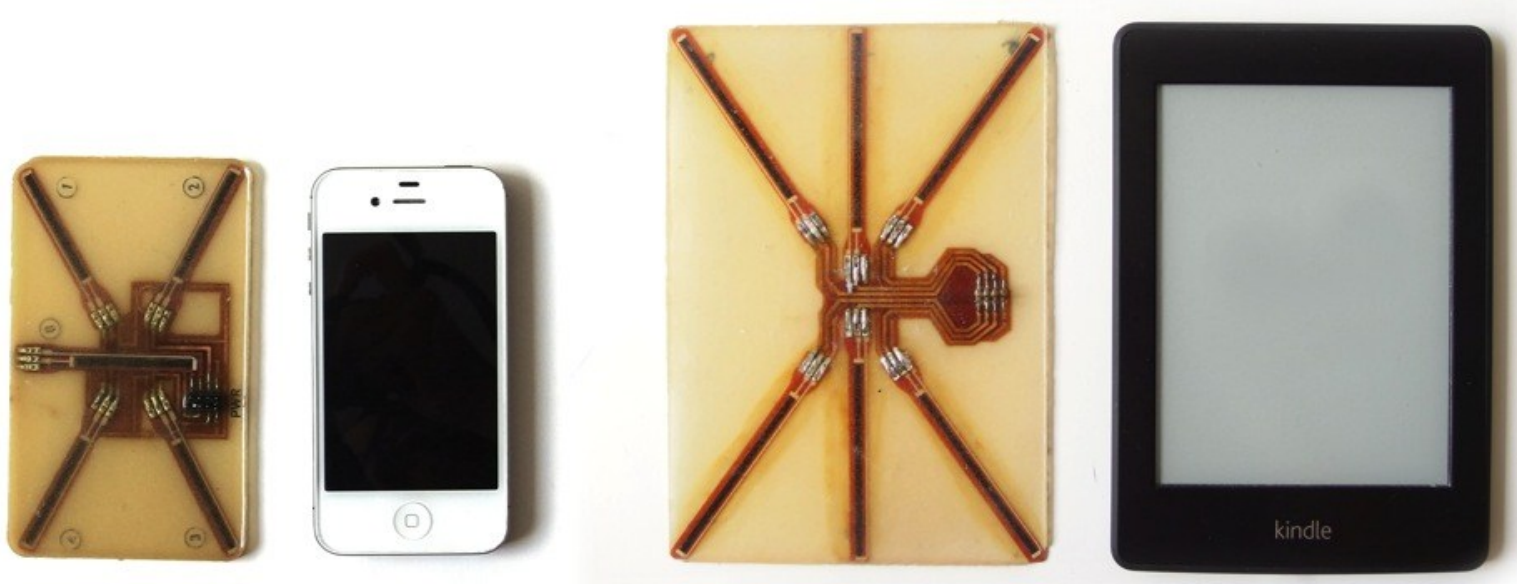

Figure 6 Small and large Bendy prototypes compared to mobile devices. 


\subsection{Bend Gesture Interaction Language}

Lahey et al. [24] define bend gestures as the "physical, manual deformation of a display surface to form a curvature for the purpose of triggering an action on a computer display". Our interaction language uses the bend gesture classification scheme proposed by Warren et al. [42] involving location and direction. Gestures can be performed in ten locations using the four corners, four sides or along the $\mathrm{x}$ or $\mathrm{y}$-axis in both directions resulting in a set of twenty gestures (Figure 7). The nomenclature used in this paper to describe gestures first states the location and secondly the direction. The four corners are described using top-right, bottom-right, top-left or bottom-left corner. Side bends are performed using the top, bottom, left or right side. Symmetrical bends are described by the parallel axis of which they are bent. The up direction indicates a gesture performed towards the user and the down direction is performed away from the user.

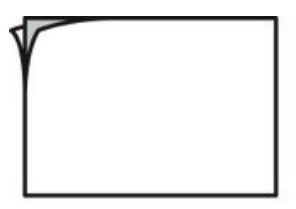

G1 Top left crnr up G2 Top left crnr down
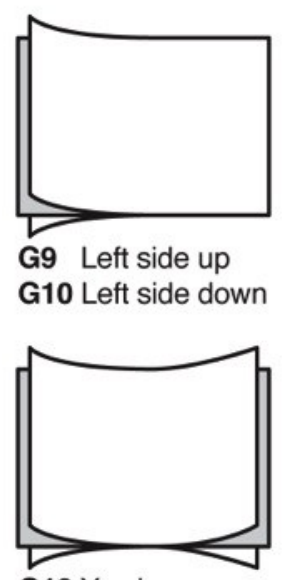

G13 Y-axis up

G14 Y-axis down

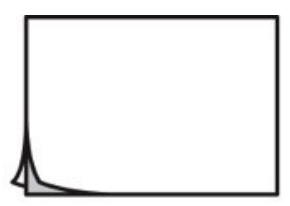

G3 Btm left crnr up G4 Btm left crnr down

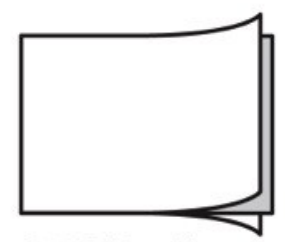

G11 Right side up G12 Right side down

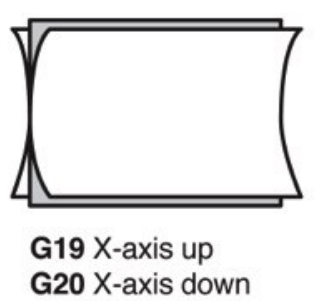

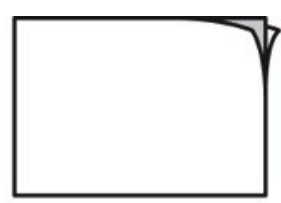

G5 Top right crnr up G6 Top right crnr down

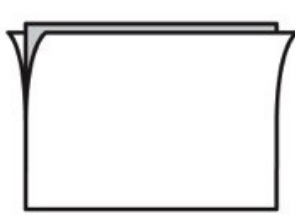

G15 Top side up G16 Top side down

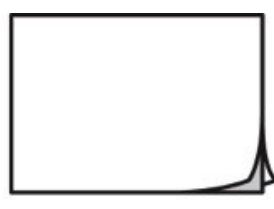

G7 Btm right crnr up G8 Btm right crnr down

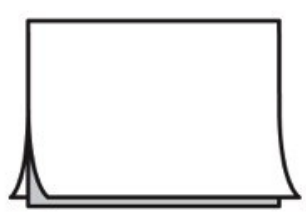

G17 Btm side up G18 Btm side down

Figure 7 Bendy's bend gesture interaction language. 


\subsection{Hardware}

The design of the hardware went through an extensive, iterative process to include the physical affordances of a real product, enable interactivity and ensure robustness as it would be subject to prolonged use. The prototypes consist of a thin piece of polycarbonate plastic backed in a thin layer of silicon resin. There are three constructive layers of the prototype: a surface substrate, the flexible circuit and silicon used to enclose the sensors (Figure 8).

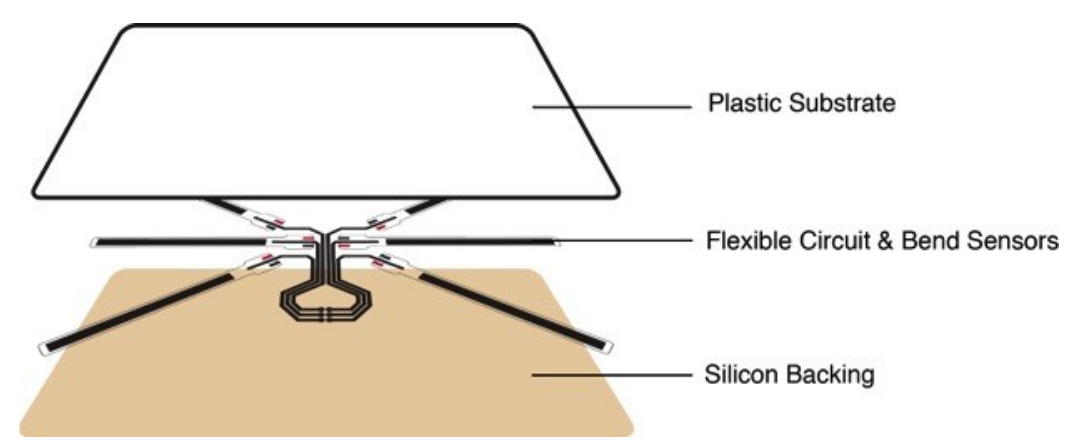

Figure 8 Exploded view of the Bendy prototype.

The first design decision was to determine an appropriate stiffness for the prototype. We used Kildal et al.'s [23] finding, which indicated that stiffer materials were least preferred by users and consequently incorporated less-stiff materials in our design. Silicon resin is available in different shore hardnesses, which is the measure of the material's resistance to permanent indentation. It is represented in a scale number ( 0 to 100) and letter (variable stiffness of plastic). We experimented with $70 \mathrm{~A}, 60 \mathrm{~A}$ and $30 \mathrm{~A}$ resins with 70A being the stiffest. A negative release mold was machine milled to size to cast the prototypes. We performed gestures on the mock prototypes and found that the $70 \mathrm{~A}$ and $60 \mathrm{~A}$ prototypes provided enough rigidity, however, they were difficult to use. Accordingly, we selected to use 30A silicon resin for our final prototype. 

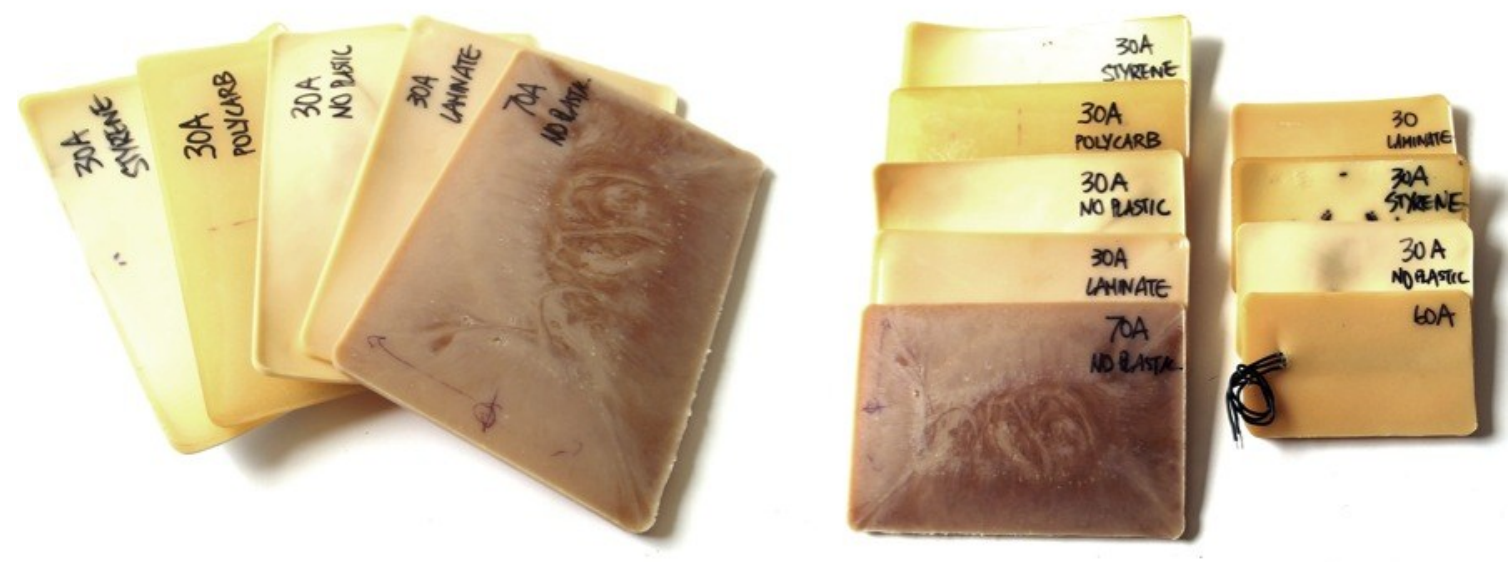

Figure 9 Iterative prototypes using different material stiffness and compositions

Once the material stiffness was decided, we needed to determine a construction method. Our first trial directly submerged the circuit into the silicon resin. It was difficult and tedious to accurately place the circuit due to the limited working time of the resin. Consequently, we used a thin piece of plastic to support the circuit. While this improved the fabrication process, the surface finish of the silicon contained ripples and air capsules. Alternatively, we cast the silicon on top of the substrate allowing for a smooth surface finish on both sides of the prototype. We experimented with polystyrene and polycarbonate for the substrate layer. Polystyrene provided the desirable rigidity but prolonged use of the prototype caused permanent deformation. Conversely, polycarbonate is a more rigid plastic and retains its shape after extended trials. Therefore, the final prototype consists of a thin piece of polycarbonate and 30A silicon resin. 


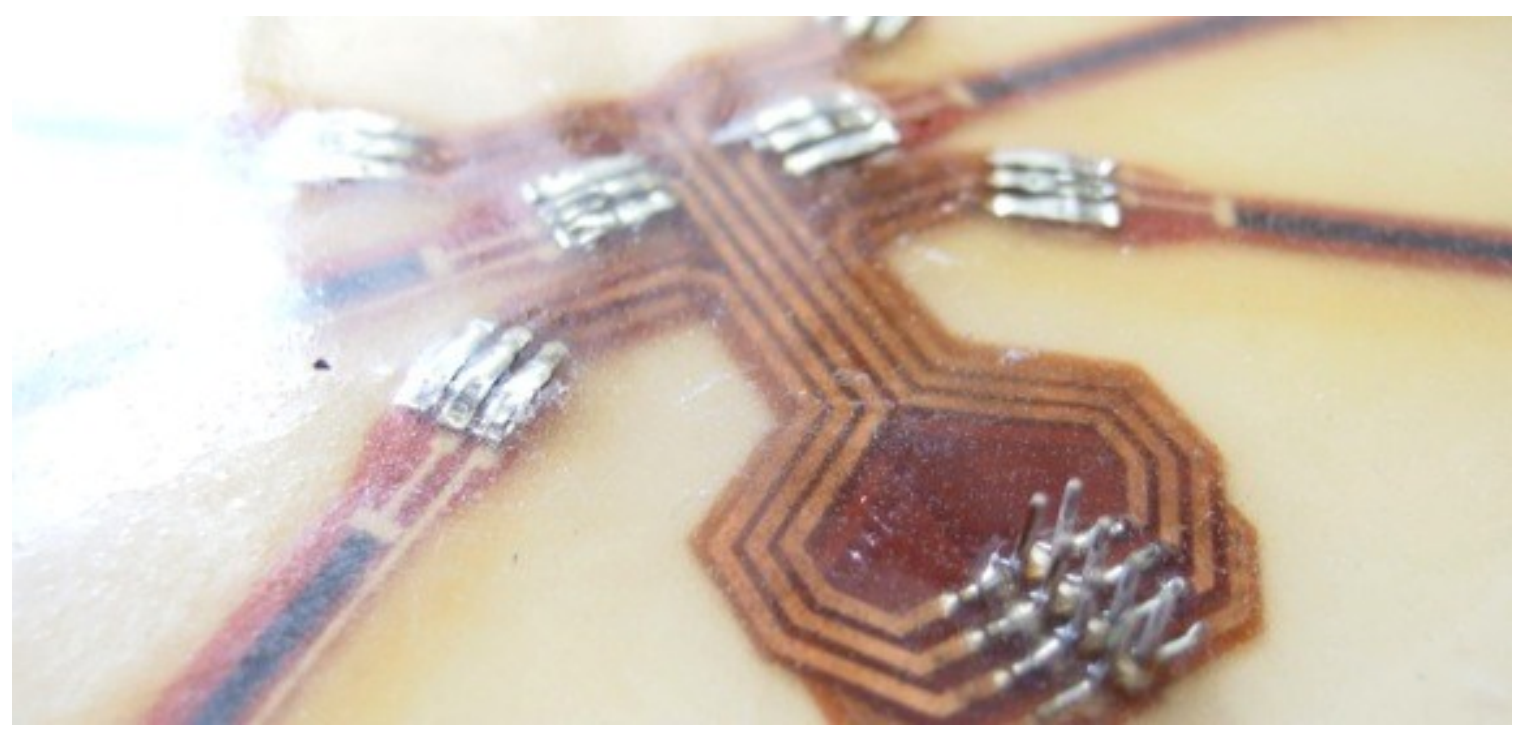

Figure 10 Flexible printed circuit

An essential component for a successful prototype was ensuring flexibility and robustness. The prototype was augmented with a flexible printed circuit (FPC) connected to six bidirectional bend sensors. We printed the circuit onto a single-sided DuPont Pyralux flexible copper sheet using a wax based ink printer. The ink protects the conductive paths, as it is resistive to copper etchant. The copper was etched using a two to one ratio of hydrogen peroxide and hydrochloric acid. After the copper was dissolved, the ink was removed using isopropanol. The stress points of the device were tested rigorously and several techniques were used to ensure that the prototype remained robust after prolonged use. As a result, the circuit paths were soldered on top of the bend sensor contacts points. The connectors were placed in the center of the device where the least amount of stress occurs. Lastly, the circuit diagram was drawn void of any right angles. 


\subsection{Software}

The prototype is connected to an Arduino Uno Microcontroller [1] using the six analog pins augmented with a calibration button. An Arduino program was written to receive and process the raw bend sensor data and calibration button. A digital smoothing algorithm was implemented to remove any outliers from the raw data. The smoothed data was then mapped to a range of 0 to 100 . Activation threshold values were set for the up and down directions. The top bends required larger thresholds than the down bends due to the resistance inherited from the material composition of the prototype. Bend gesture output was programmed by the activation of the sensors. For example, if sensor 2 and sensor 3 were both activated upwards this resulted in gesture 15. All gesture recognized were discrete, that is if a top-right-corner up was bent simultaneously with the top-leftcorner up, the gesture would be recognized as a top-side up bend. When the device is in neutral position, pressing of the button set the threshold values. This allowed for the devices to be easily calibrated. The bend gesture output is programmed into the Processing applications used for gaming. Daniel McCarney assisted with the creation of the bend gesture recognition program. We used the open-source processing community forum (www.openprocessing.org) to assist with the development of the games. The games were altered to fit our research needs. 


\subsection{Apparatus}

An Optoma pico-projector (PK301) [30] was used to simulate the visual display. We made adjustments before and in between each session to focus the display. A fiducial marker was placed on the back of the prototype and a web camera was positioned behind to detect its location using the reacTIVision [21] software. We integrated this position data in the Processing applications to keep the projection within the frame of the prototype. The Bendy set up is illustrated in Figure 11.

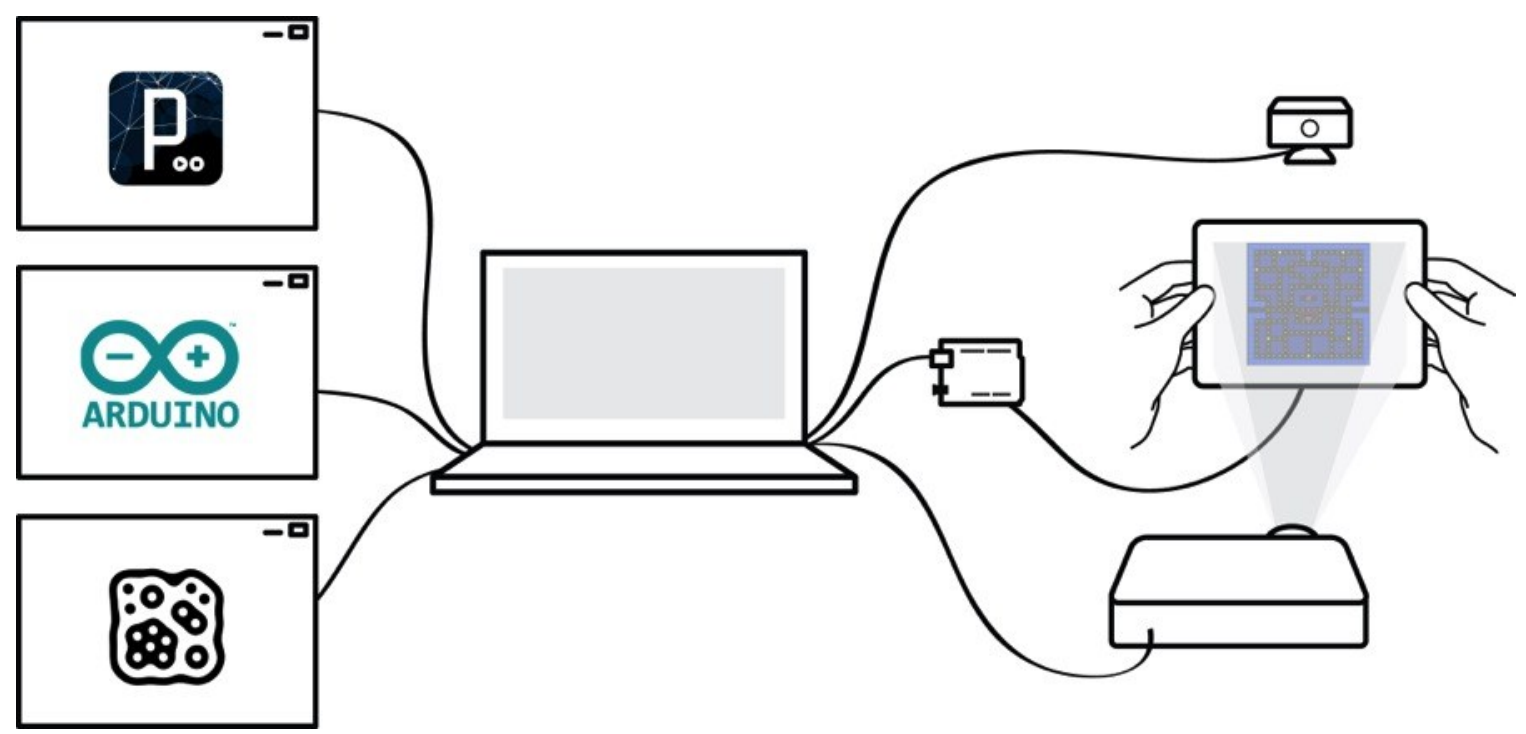

Figure 11 Bendy apparatus 


\section{Chapter: Experiment 1: User-Defined Bend Gestures for Gaming}

\subsection{Overview}

Bend gestures are a novel interaction language for users. As a result, there are many intricacies to be examined, especially in the context of mobile gaming. How will users map game controls? How will users translate physical concepts to bend gestures? Our main research goal is to explore these intricacies and generate user-defined bend gestures for gaming. We conducted a participatory experiment using Wobbrock et al.'s [45] guessability methodology. This methodology proposes to observe user behaviour given the effects, the visual feedback of the system, and ask users to create the cause, the bend gesture. This process creates a dialogue between the system and the behaviour enabling users to provide accurate response selections. In addition, a participatory experiment facilitates the observation of users and allows researchers to gain insight into users' behavioural patterns and mental models. Wobbrock's study generated user-defined gestures, which performed significantly better compared to designer-defined gestures. Lahey et al. [24] and Lee et al. [27] applied this methodology in the context of deformable user interfaces. In addition to our main research goal, we test the Simon Effect by observing if the location of the stimulus would have an effect on the performed gesture location.

Our secondary research goal was to examine if there were differences in user performance when using different sized devices. Research indicates that there is a preference for small size (handheld) versus large size (A4), but studies have not compared sizes commonly found in mobile devices, such as smartphones and small computing tablets. 


\subsection{Tasks}

We proposed a set of nine tasks derived from simple game commands. The tasks are grouped into three categories: translational, action-specific and physical manipulations. Each task is divided into unique events. Figure 12 illustrates the nine tasks and thirty-seven events. We presented participants with a 2D visual stimulus using simple shapes and recorded their bend gestures.

Participants were required to map gestures to several events within each task. We used multiple events per task to observe how participants would map gestures given a group of stimuli without collocation. For example, in the shooting task participants were asked to select three unique bend gestures for three events: to trigger shooting, translating left, and translating right.

The translational tasks were selected to test patterns for the Simon Effect. Our research goal was to observe if location of the stimulus would have an effect on the bend gesture performed. For the $\mathrm{x}$-axis translation, the stimulus was presented in three locations: top, center and bottom. The y-axis translation placed the stimulus in the left, center and right location. Hereinto, events are described according to, first, the event and then the location of the stimuli (e.g. up-left is the up event in the left location). Within the $\mathrm{x}$-axis and $\mathrm{y}$-axis tasks participants were instructed that they could select gestures independently for each event. The XY translation task was selected to observe how participants would map four events to gestures given the condition that bend gestures had to remain unique.

These $\mathrm{x}$ and $\mathrm{y}$-axis events were selected to test for patterns that would indicate the presence or absence of the Simon Effect. We hypothesized that participants would select 
gestures according to the location of the stimulus (H1). For example, when the location is presented on the left, participants will perform a bend gesture using the left side of the device.

For the action-specific tasks, we selected three actions commonly found in gaming: shooting, jumping and rotating clockwise. In addition to each action-specific event, participants were required to create separate bend gestures to translate left and right, for a total of three events per task. We hypothesized that participants will have low consensus in transforming these tasks into bend gestures, and more specifically, in the rotating task (H2). Prior works have indicated users tend to perform gestures in close relation to real-world actions, as it would be more intuitive to rotate the device rather than bend the device.

The physical manipulation tasks were selected to explore possible novel interactions leveraging the properties of the flexible device. We devised a spring task where an object was pulled back and sprung forward by release. Participants were also required to assign gestures for up and down translation of the object. The elasticity task explored compressing and stretching an object along different axes. The final task asked participants to manipulate the magnitude by increasing and decreasing the size of an object. We hypothesized that these three tasks would achieve a higher level of consensus due to their relative closeness to real-world actions (H3). 
Translations

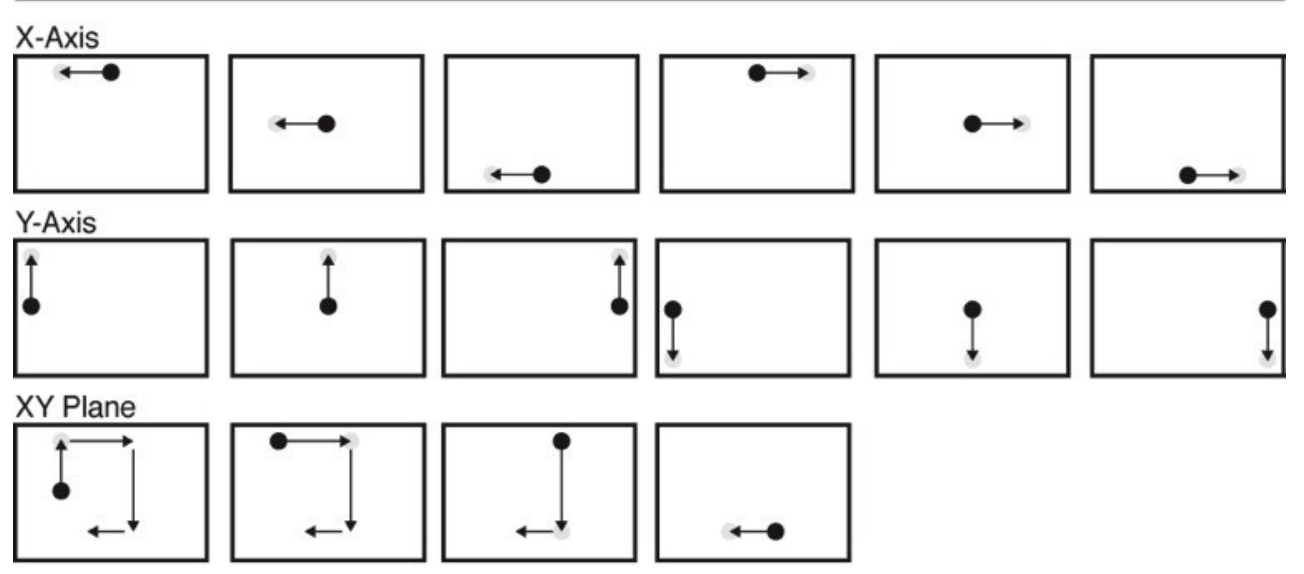

Action-Specific

Rotate

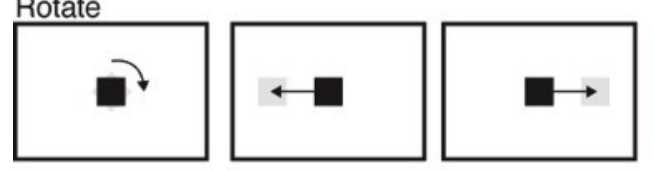

Shoot

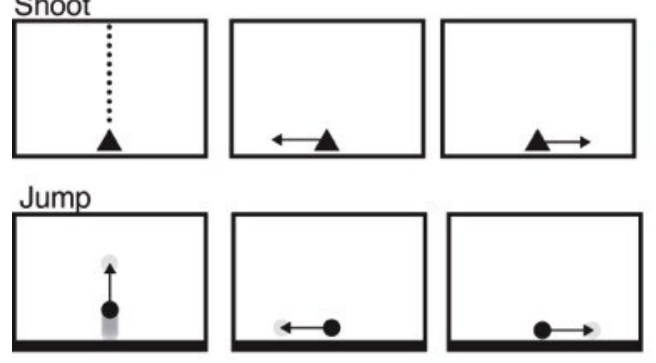

Physical Manipulations

Spring

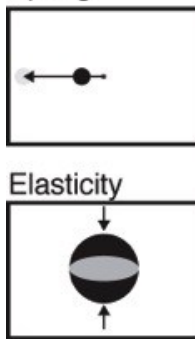

Magnitude

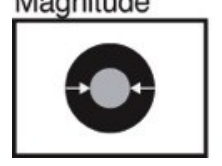

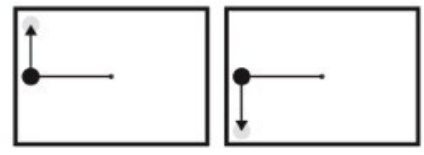
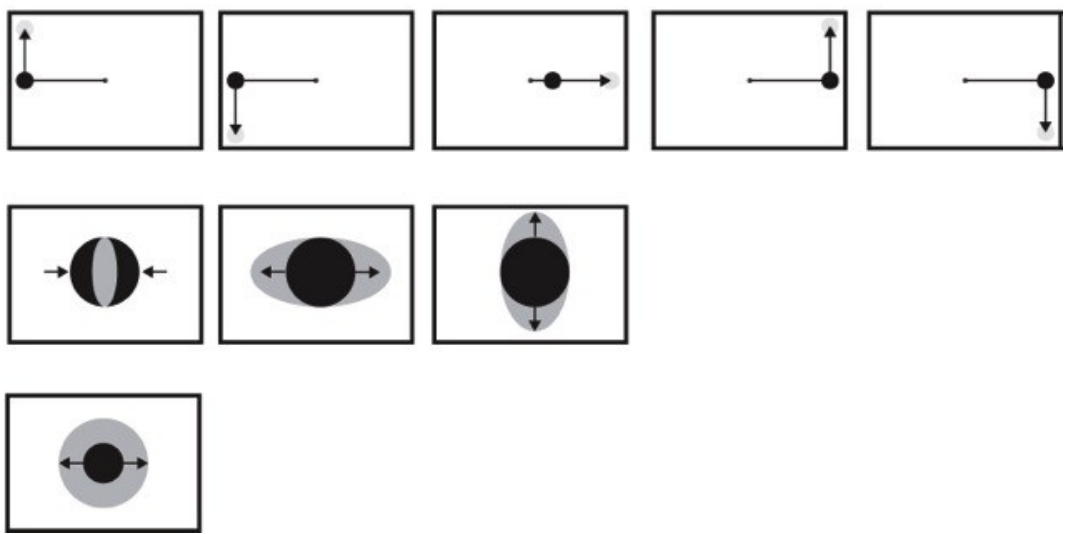

Figure 12 Animations of the tasks. 


\subsection{Participants}

Our 24 participants (15 female) had an average age of 27.2 years. One participant was left-handed. Participants had no prior experience gaming with bend gestures. Participants on average reported playing games occasionally (3.63 on a 7 point scale) and had average gaming experience (3.54 on a 7 point scale). Participants were given $\$ 10$ compensation.

\subsection{Methods}

The study was conducted in the observation lab at Carleton University. The sessions were approximately thirty to forty-five minutes. Participants were given a brief introduction to the experiment as well as a demonstration of the twenty bend gestures they could perform. The software presented the nine tasks randomly, and events were randomly presented within the translational and physical manipulation tasks. After viewing the visual stimuli for each event, participants were asked to respond with a bend gesture. The researcher asked participants to repeat the bend gestures to confirm their decisions. The procedure was repeated for both prototype sizes in a counterbalanced order. Participants were instructed that their previous choice of bend gestures did not need to remain consistent between tasks or device sizes, and that conditions were independent of each other.

\subsection{Results}

Each participant contributed 37 gestures for each size of the prototypes for a total of 1,776 gestures (Figure 14). From the collected bend gestures, we calculated an agreement score and created user-defined gesture sets. The bend gesture with the largest frequency was chosen for each event. We also identified events with conflicting results. 
We define conflict as gestures that occur in equal frequencies or received a low agreement score. Figure 15 illustrates the user-defined gesture sets. We computed an agreement score [46] for each event, which represents the degree of consensus amongst participants. The value calculated varies between 0 and 1 , with a higher number indicating a stronger agreement. The formula for calculating the agreement score is:

$$
A_{e}=\sum_{P_{i}}\left(\frac{\left|P_{i}\right|}{\left|P_{e}\right|}\right)^{2}
$$

where $e$ is an event in the set for all events $E ; \mathrm{P}_{i}$ is the set of proposed bend gestures for $e$; and $\mathrm{P}_{i}$ is a subset of idential gestures for $\mathrm{P}_{e} . \mathrm{A}_{e}$ is a number between 0 and 1 . For example for the event translate object left, of the 24 partcipants, 15 choose left-side-down (G10), 7 choose right-side-up (G11), 1 participant choose the bend gesture top-left-corner-down (G2) and 1 participant choose top-right-corner-up (G7). The agreement score for this event is:

$$
A_{\text {left }}=\left(\frac{15}{24}\right)^{2}+\left(\frac{7}{24}\right)^{2}+\left(\frac{1}{24}\right)^{2}+\left(\frac{1}{24}\right)^{2}=0.48
$$

The agreement scores are graphed in descending order for both size small and large (Figure 13). In both sizes, the physical manipulation events ranked the highest, whereas the action-specific tasks ranked the lowest. The translation events in the action-specific tasks were omitted from our analysis to remove data overlap with the translational tasks. 
Small

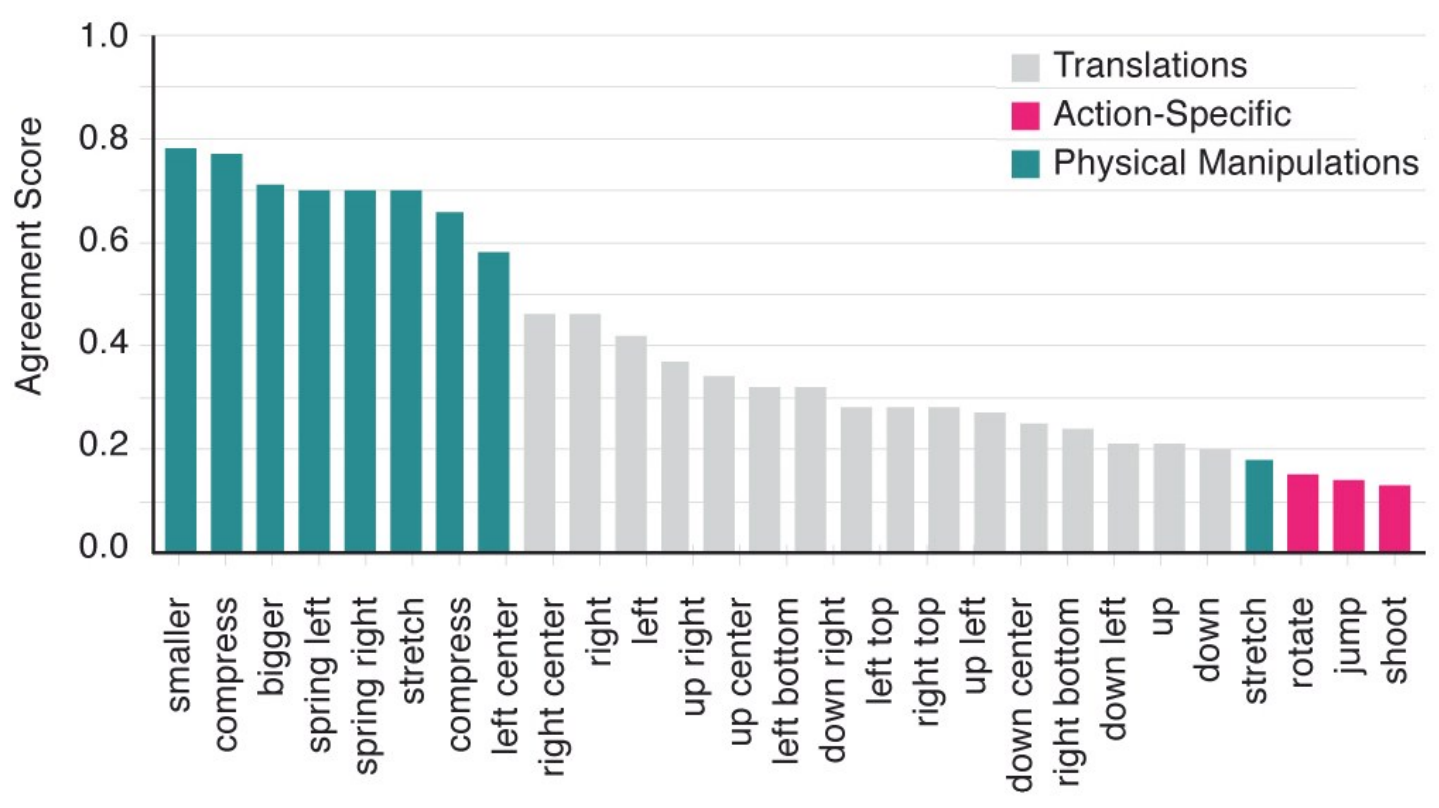

Large

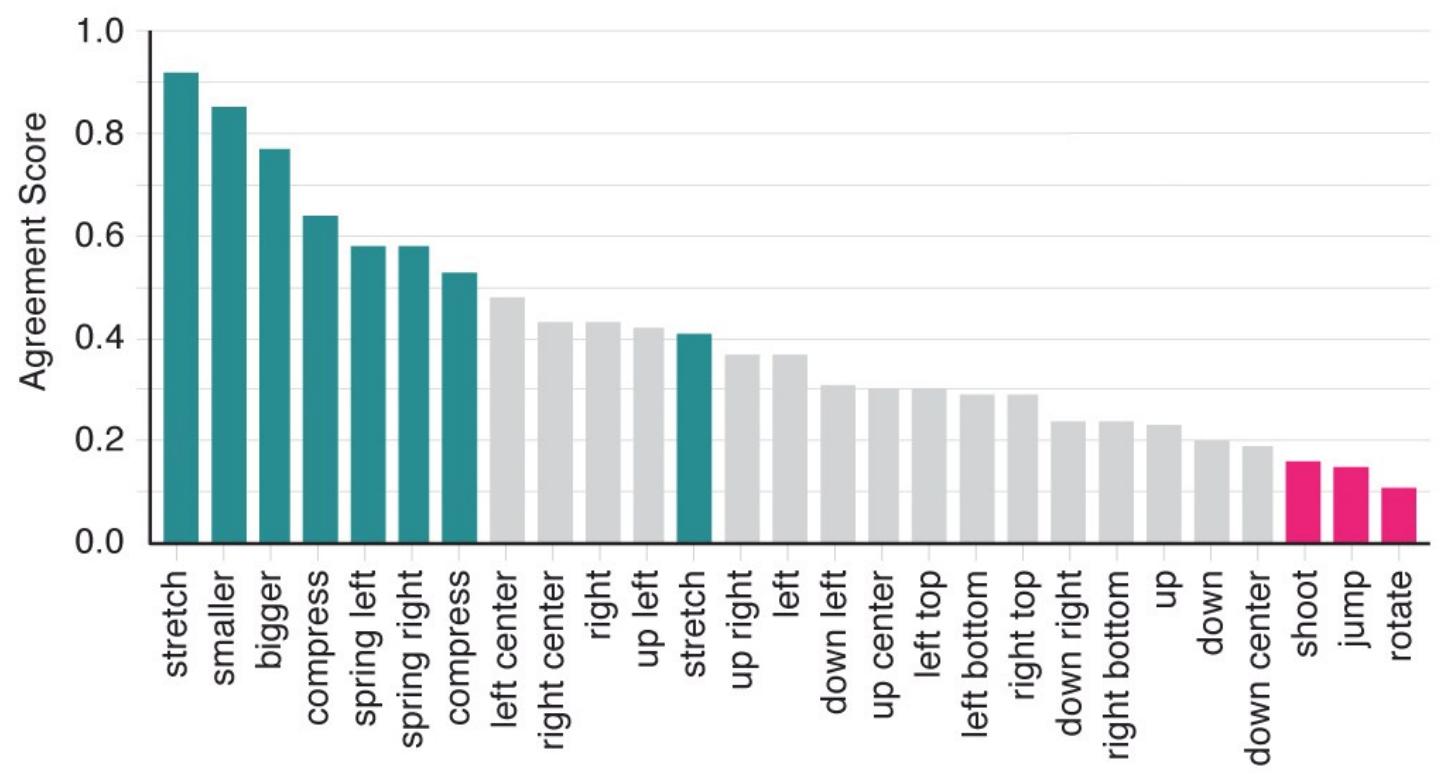

Figure 13 Agreement scores for both device sizes. 

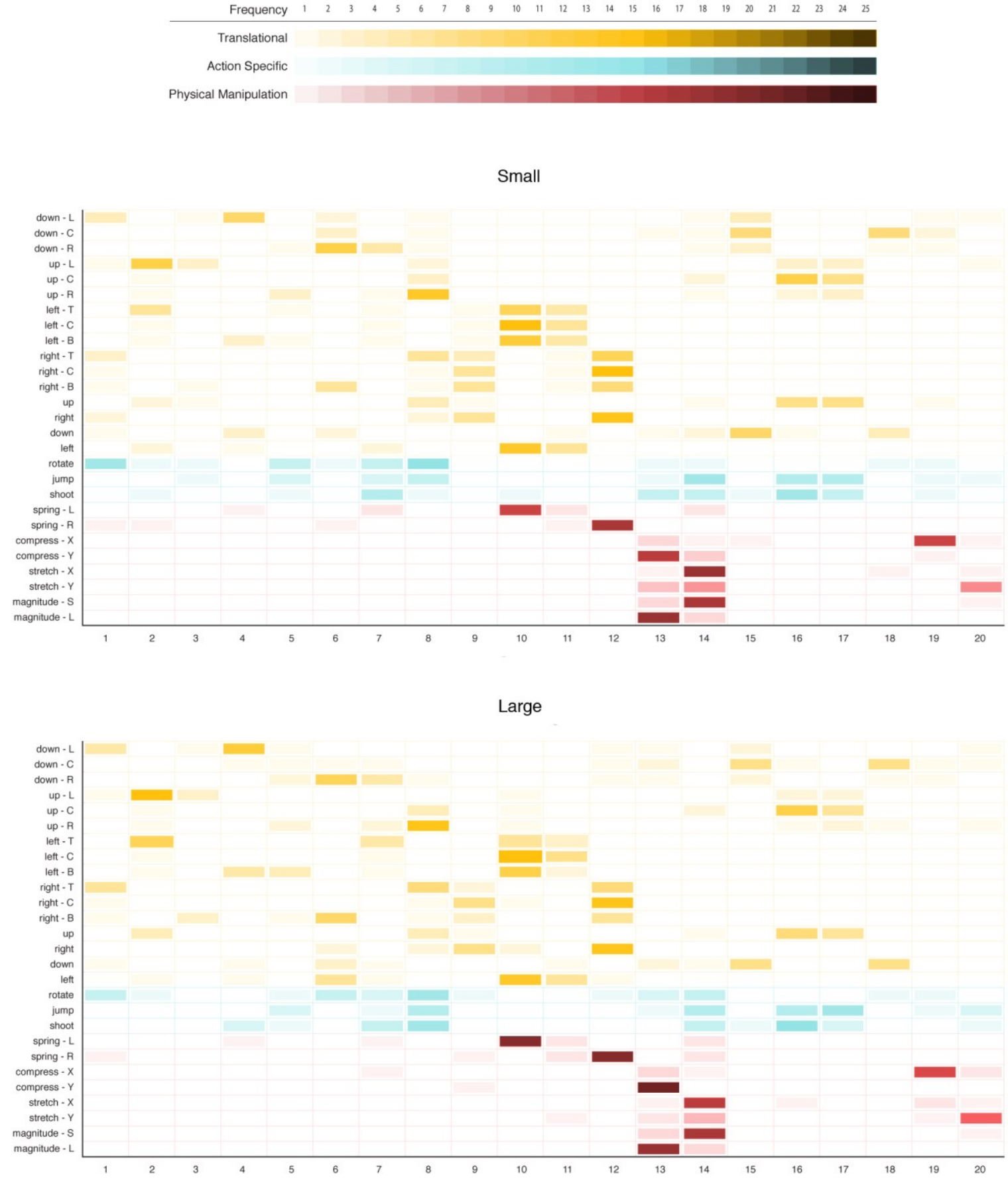

Figure 14 Frequency plot of the gestures collected. 


\subsubsection{Translation Tasks}

\subsubsection{Y-Axis Translation}

The gesture sets are the same for both sizes. If the stimulus was located on the left, participants chose to use the top-left-corner-down (G2) and bottom-left-corner-down (G4) to perform the event up and down, respectively. Similarly, when the location of the stimulus was on the right, participants chose to use the top-right-corner-down (G8) and bottom-right-corner-down (G6). When the stimulus was presented in the center location, participants chose top-side-down (G16) to perform the up event. A conflict occurred in both sizes for the event down-center. Participants either performed the gesture top-sideup (G15) or bottom-side-down (G18). The conflict occurs as participants paired opposing events equally by either location or direction.

To evaluate the Simon Effect, gestures performed were compiled according to location: left, center and right (Table 1). A Chi-square test for association was conducted between the location of the gesture performed and visual stimulus location. All expected cell frequencies were greater than five. There was a significant association between the location of the gesture performed and visual stimulus location for both sizes ( $\operatorname{small} \chi^{2}(4)$ $=108.677, \mathrm{p}<.001$ and large $\left.\chi^{2}(4)=138.739, \mathrm{p}<.001\right)$. For both size devices the strong association indicates participants performed the gestures in the location of the visual stimuli. 
Small Size

\begin{tabular}{|c|c|c|c|c|c|c|c|}
\hline \multicolumn{4}{|l|}{ 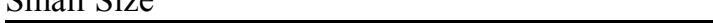 } & \\
\hline Gesture Location & \multicolumn{3}{|c|}{ Visual Location } & Gesture Location & \multicolumn{3}{|c|}{ Visual Location } \\
\hline & Left & Center & Right & & Left & Center & Right \\
\hline Left & 29 & 1 & 1 & Left & 38 & 3 & 1 \\
\hline Center & 14 & 40 & 12 & Center & 8 & 38 & 10 \\
\hline Right & 5 & 7 & 35 & Right & 2 & 7 & 37 \\
\hline
\end{tabular}

\subsubsection{X-Axis Translation}

For the $\mathrm{x}$-axis translation on the small device, participants selected left-side-down (G10) and right-side-down (G12) to perform the events, left and right, respectively. The location of the visual stimuli did not affect the gesture location.

For the left event on the larger device, participants selected left-side-down (G10) when the stimulus was presented in the bottom and center location. For the top visual location they selected the top-left-corner-down (G2). For the right event, participants selected bottom-right-corner-down (G6) when the stimulus was presented on the bottom and, for the center visual location, right-side-down (G12). A conflict occurred for the right event in the top visual location. Participants equally selected top-right-corner-down (G8) or right-side-down (G12).

Similar to the y-axis translation task, the bend gestures performed were compiled according to location: top, center and bottom (Table 2). A Chi-square test for association was conducted between the location of the gesture performed and visual stimulus location. All expected cell frequencies were greater than five. There was a significant association between the location of the gesture performed and visual stimulus location for both sizes (small $\chi^{2}(4)=33.222, p<.001$ and large $\left.\chi^{2}(4)=90.937, p<.001\right)$. For the large device, there is a stronger association between gesture location and visual location. 
On the small device, there is a greater tendency to perform center gestures regardless of visual location.

Small

\begin{tabular}{r|cccc}
\hline $\begin{array}{r}\text { Gesture } \\
\text { Location }\end{array}$ & \multicolumn{4}{|c}{ Visual Location } \\
\hline & Top & Center & Bottom & Total \\
Top & 16 & 4 & 4 & $24(17 \%)$ \\
Center & $\mathbf{3 1}$ & $\mathbf{4 4}$ & $\mathbf{3 3}$ & $\mathbf{1 0 8}(\mathbf{7 5 \%})$ \\
Bottom & 1 & 0 & 11 & $12(8 \%)$ \\
\hline
\end{tabular}

Large

\begin{tabular}{r|cccc}
\hline $\begin{array}{r}\text { Gesture } \\
\text { Location }\end{array}$ & \multicolumn{4}{|c}{ Visual Location } \\
\hline & Top & Center & Bottom & Total \\
Top & $\mathbf{2 9}$ & 4 & 4 & $37(26 \%)$ \\
Center & 19 & $\mathbf{4 4}$ & $\mathbf{2 2}$ & $\mathbf{8 5}(\mathbf{5 9 \%})$ \\
Bottom & 0 & 0 & $\mathbf{2 2}$ & $22(15 \%)$ \\
\hline
\end{tabular}

Table 2 Gestures performed for the $x$-axis translation for both sizes. 


\subsubsection{XY Plane Translation}

The bend gestures for the left and right events are left-side-down (G10) and rightside-down (G12) for both sizes. For the small device, participants selected top-side-down (G15) for the down event. On the large device, participants selected top-side-down (G16) for the up event. A conflict similar to that found in the y-axis translation task also occurred in this task. The conflict occurred on the small device where both top-side-down (G16) and bottom-side-up (G17) result in equal frequency for the event up. The second conflict occurs in the large device, where top-side-up (G15) and bottom-side-down (G18) also occur in equal frequency for the event down. For both sizes, participants showed conflicting behavior by choosing to pair the event using either location or direction.

\subsubsection{Action-Specific Tasks}

The agreement scores for all three action-specific tasks ranked the lowest for both devices (average of 0.14 for both sizes) and also resulted in the largest number of unique gestures performed (averages: small 10.6, large 10). There was no observed consensus amongst participants for any particular gesture. The gestures for translating left and right were left-side-down (G10) and right-side-down (G12), which is consistent with the results from the $\mathrm{x}$-axis translation task.

\subsubsection{Physical Manipulation Tasks}

\subsubsection{Spring}

For the spring task, both device sizes exhibited the same gesture-task pairings. The bend gesture chosen for the spring task was left-side-down (G10) to pull the spring to the left, top-left-corner-down (G2) to move it up and the bottom-left-corner-down $(\mathrm{G} 4)$ to move it down. 


\subsubsection{Elasticity}

For the compression events, participants selected x-axis-up (G19) to compress an object along the y-axis and the y-axis-up (G13) to compress along the x-axis. Bend gestures for compression were performed in the up direction, and bend gestures for stretch were, conversely, performed in the down direction. Participants selected y-axisdown (G14) to stretch along the $\mathrm{x}$-axis and $\mathrm{x}$-axis-down $(\mathrm{G} 20)$ to stretch along the $\mathrm{y}$-axis. However, the stretch event along the y-axis received a low agreement score value $(0.18)$ on the small device as 10 participants performed the $\mathrm{x}$-axis down and 9 participants performed y-axis down.

\subsubsection{Magnitude}

In both sizes, the bend gestures for increasing and decreasing magnitude were yaxis-down (G14) and y-axis-up (G13), respectively.

\subsection{Post Experiment}

In the first experiment, five events did not reach a consensus. The conflicts were down-center, right-top, jump, shoot and rotate. We conducted a post experiment to resolve these conflicts where only the most frequent bend gestures were proposed to participants. Table 3 is a summary of the five conflicts and the bend gestures we chose to re-test.

\begin{tabular}{l|l}
\hline Event & Bend Gestures \\
\hline Down (center) & Top-side-down (G15) and bottom-side-down (G18) \\
Right (top) & Right-side-down (G12) and top-right-corner-down (G8) \\
Jump & Y-axis down (G14), bottom-side-up (G17) and top-side-down (G16) \\
Shoot & Top-right-corner-up (G7), top-right-corner-down (G8) and top-side-down (G16) \\
Rotate & Top-left-corner-up (G1) and top-right-corner-down (G8) \\
\hline
\end{tabular}

Table 3 Unresolved gesture conflicts in the first experiment. 


\subsubsection{Participants}

12 participants were recruited for the experiment (6 female). Participants did not participate in the first experiment and had no prior experience gaming with bend gestures. Participants were on average 22.66 years old. All participants were right-handed, reported playing games occasionally (3.08/7) and had an average gaming experience. (3.83/7). Participants were given $\$ 10$ compensation.

\subsubsection{Procedure}

Participants were given the same visual stimuli as in the previous experiment, and asked to respond with a bend gesture. If the participants did not perform a candidate gesture, they were provided with the options and asked to choose one. We presented the events randomly. We conducted the experiment using only the large device because no meaningful differences were observed between the sizes in the first experiment.

\subsubsection{Results}

A consensus was found for only 2 of the 5 events. 10 participants chose rightside-down (G12) for the right-top event. 9 participants selected the bottom-side-up (G17) for the jump event. The other 3 events were left unresolved, as participants did not reach a consensus. For example in the shooting event, 7 participants selected top-right-cornerdown (G8) and 5 participants selected top-left-corner-up (G1). 

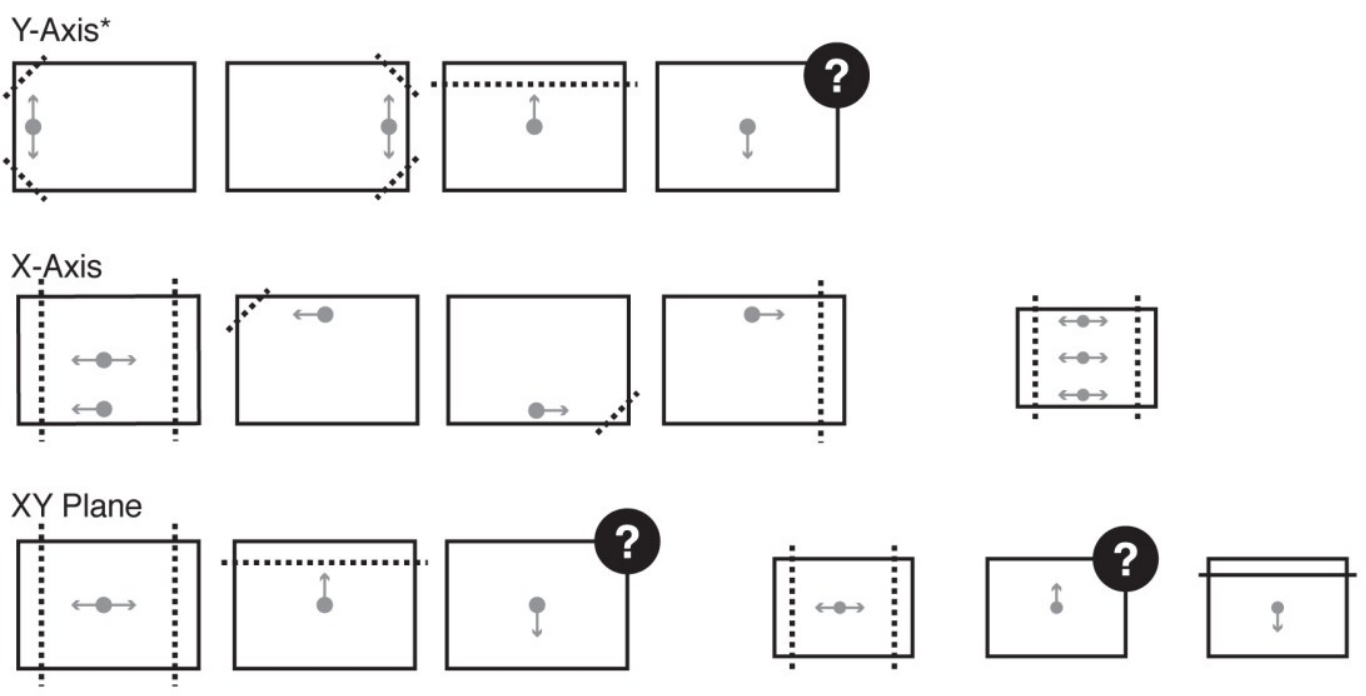

\section{Action-Specific}

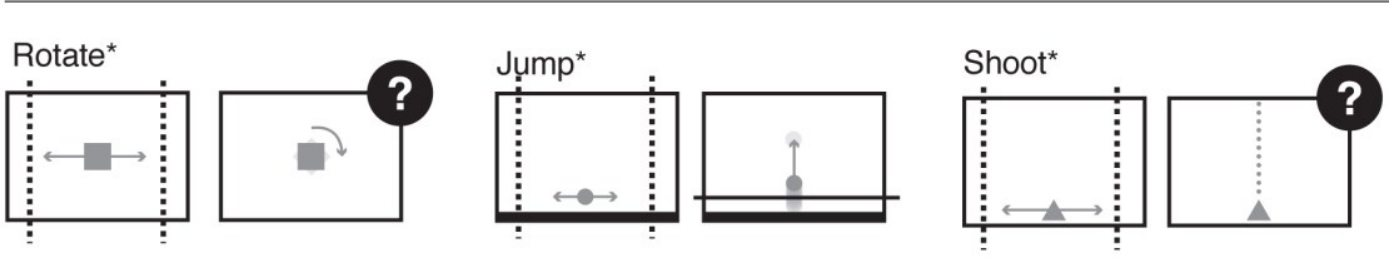

\section{Physical Manipulations}
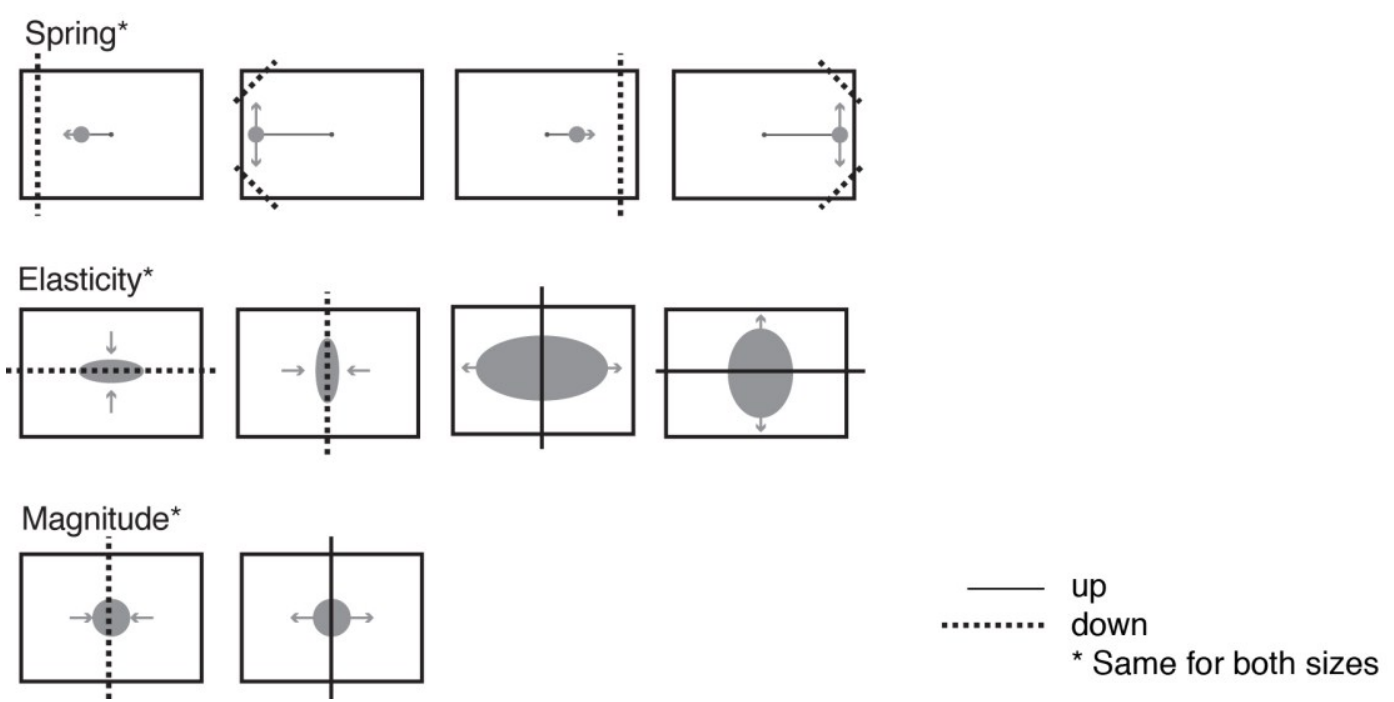

Figure 15 User-defined gesture sets including unresolved conflicts. 


\subsection{Discussion}

Our results show that there is a general consensus among users when selecting gestures for most of the tasks. We report our insights gathered from the quantitative analyses and observations. Our experiment found novel interaction paradigms including user mental models, behaviour and performance.

\subsubsection{User Consensus}

As shown in Figure 13, physical manipulation tasks ranked the highest in user agreement $(\mathrm{H} 3)$ and had the lowest average of unique gestures performed (small: 3.6 gestures, large: 4.0). Participants found it easier to create gestures for these tasks. Even for our participants who had no previous experience interacting with flexible devices, creating gestures was perceived to be effortless. For users pulling the device downward translated easily into increasing magnitude or stretching the object. Pushing the device upward was translated inversely, decreasing magnitude or compressing an object. The actions performed for these tasks leverage real-world actions such as squeezing a ball to make it smaller or stretching a rubber band to make it larger.

The action-specific tasks ranked the lowest in user agreement (H2), with the highest average of unique gestures performed (small: 10.6 gestures, large: 10.0). During the shooting and jumping tasks, some participants derived their gestures based on a perceived direction for the action as indicated by responses such as "Because I'm shooting in that [up] direction" or by stating "Because I'm jumping up". Participants found the in plane rotation task difficult to conceptualize as a gesture input. We observed an immediate inclination to rotate the device. To help formulate a bend gesture participants often asked if the direction of the object would change or remain in 
clockwise rotation. In many cases, participants selected the top-right-corner solely for ease of use. The case may be different for out of plane rotation. Participants reported associating the use of the top corners of the device with the act of manipulating game console controllers, where the action buttons are often located on the right. They often reported, "I would use it like a trigger".

\subsubsection{Opposing Events with Paired Gestures}

Participants paired gestures with opposing events, a finding supported by prior work [24]. While the $\mathrm{x}$ and $\mathrm{y}$-axis events were not presented in pairs in our experiment, most participants (85\%) created paired gestures using location (e.g. the G10 and G12 combination). Only a few (4\%) chose to pair gestures by direction and it was only performed during the y-axis task. We noted that one of the conflicts found in our experiment was for the event down-center. Participants were conflicted by either pairing the event by location or by direction. In this case, while it may have been more intuitive to pair by location, participants may have found it more ergonomic to pair by direction.

\subsubsection{The "Push" or "Pull" Mental Model}

During the translation tasks, participants demonstrated two mental models that governed their perception of how to manipulate the virtual objects. They either "pushed" or "pulled" the object in the desired direction Figure 16. For instance, a participant would use the left side of the device down (G10) to "pull" the object left; whereas another participant might bend the right side up (G11) to "push" the object left. We analyzed the translational tasks and observed 56\% utilized the "pull" concept whereas $25 \%$ performed the "push" concept and the remaining 19\% showed no evidence of a push-pull mental 
model. The "push" and "pull" models correlate with the observation that participants commonly paired gestures by location.

Once participants developed their mental model of how to manipulate the virtual environment, it remained consistent throughout the experiment. Subsequently, the chosen mental model also dictated the direction of the gestures. If the participant perceived his or her actions using the "pull" concept, their gestures were more likely to be performed downwards for subsequent events. Conversely, if participants choose to follow the "push" concept, their gestures were more likely to be performed upwards.
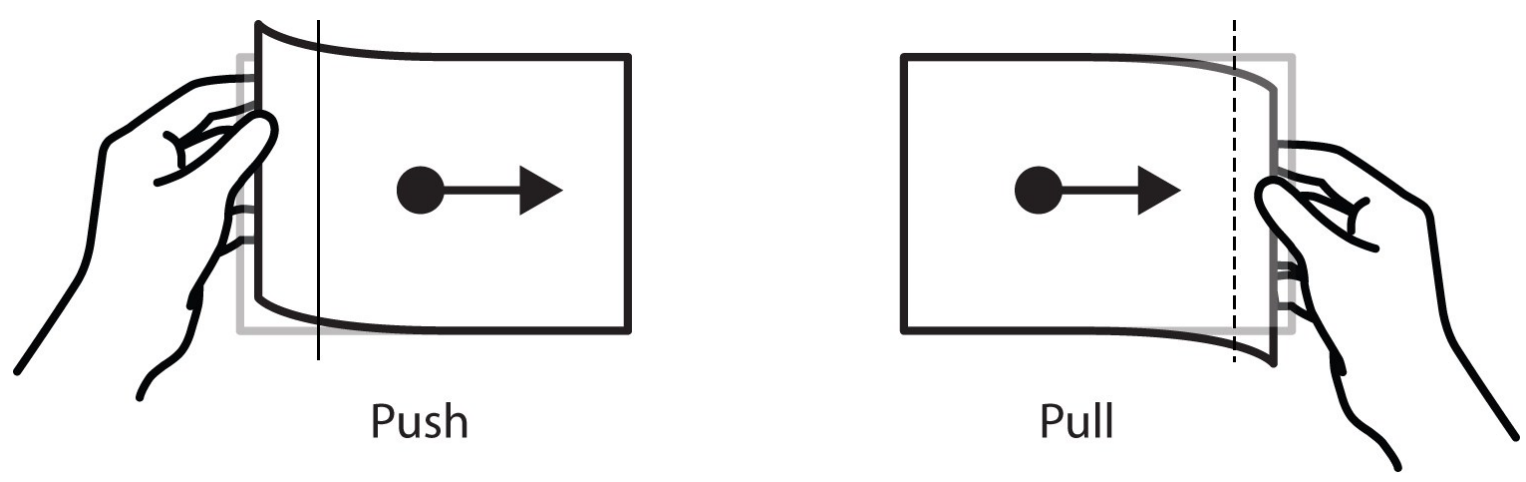

Figure 16 The "push" or "push" mental models.

\subsubsection{Consistency Among Sizes}

Our secondary research goal was to explore whether a difference in device size would affect gestures. During the experiment we instructed participants that the gestures they created for the previous size did not need to remain the same. Despite these instructions, we observed very little differences in performance between the sizes. However, a limitation to this finding is the within subjects design of the experiment. While the results suggest little difference between the gestures performed between sizes an independent groups experiment would need to be conducted to further support this 
finding. A noticeable difference is the effect of size on visual location and hand position, which will be discussed in following section.

\subsubsection{Simon Effect: Collocation of Stimulus and Gesture}

One of our main research goals was to evaluate the presence of the Simon Effect, which would link the location of the stimulus and that of the selected gesture. Our results indicate a strong association between visual stimulus locations and bend gesture location (H1). However, the patterns found manifested themselves differently according to the stimuli. In the y-axis translation task, the bend gesture was performed where the visual stimulus was located. Surprisingly, the $\mathrm{x}$-axis translation task did not follow the same pattern. The association is towards performing a bend gesture in the central location regardless of stimulus location, especially on the small device. On the large device, while there is the same strong association exists; there is also a greater distance between visual locations in the y-axis task. The gestures started to gravitate towards the corresponding visual location. This suggests that as the distance between stimuli increases, participants are more likely to perform the gesture in the location of the stimulus. Overall, our results follow the Simon Effect when the distance between the stimuli is sufficient enough, in our case, during the $\mathrm{y}$-axis translation on both sizes and $\mathrm{x}$-axis translation on the large device.

\subsubsection{Different Hand Positions}

Participants held the device in many different ways to perform gestures. Figure 17 illustrates a few common positions. The way the participants held the device also influenced how the gestures were selected. Some positions restricted the performance of 
gestures to only one direction. Changing hand positions happened most often when performing difficult bends, such as bottom-side-down (G18).
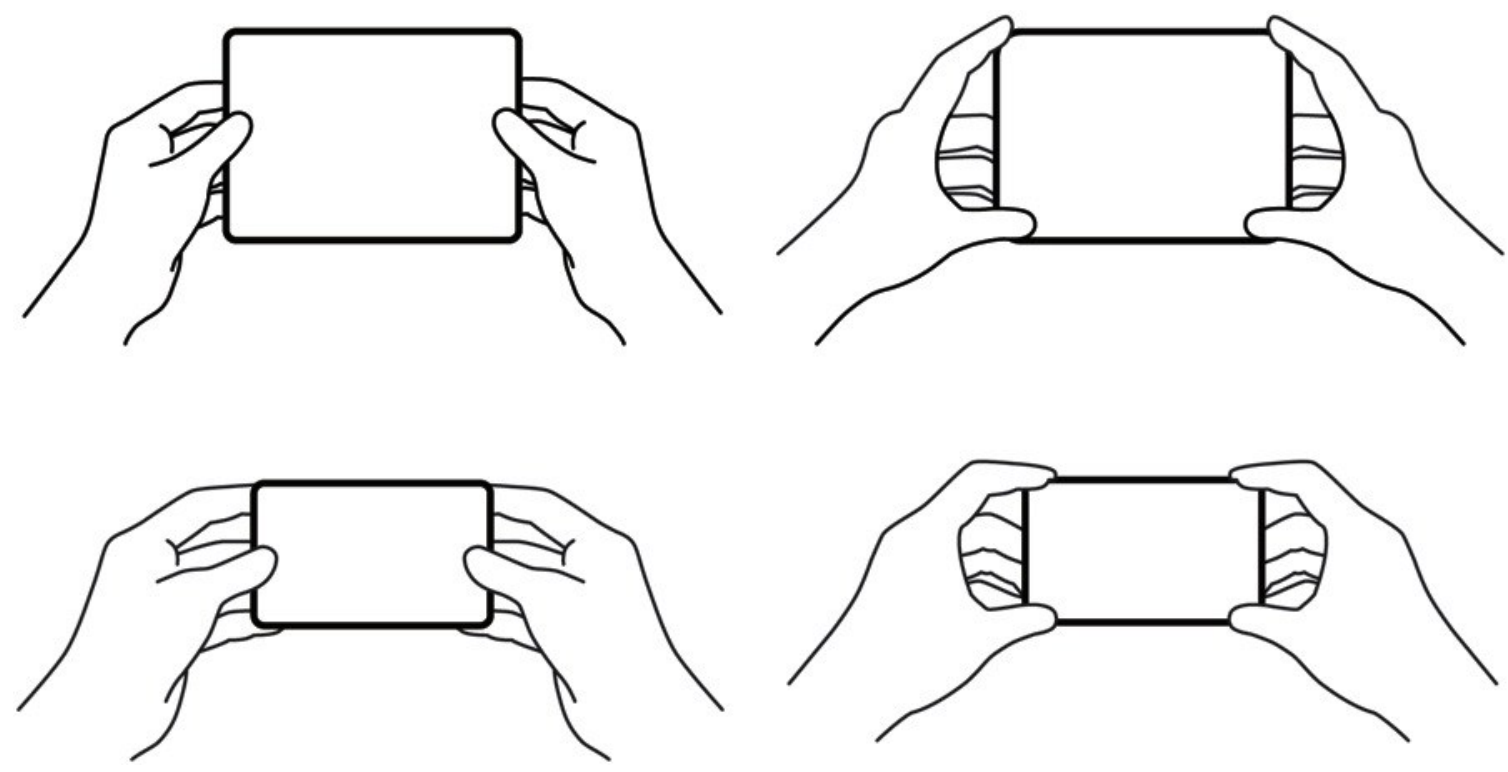

Figure 17 Illustration of how some users held the prototypes.

\subsection{Summary}

We explored gaming on mobile flexible devices with an initial participatory experiment. We deconstructed simple games into nine tasks, grouped as translation, actions-specific or physical manipulation, and asked users to define bend gestures using the guessability methodology. Our results provide a set of gestures for most of the proposed events. During our experiment, we found five sources of conflicts, which we tried to resolve in a post experiment. Unfortunately, three events still remain unresolved. Consequently, physical manipulations and translations are the tasks with the most potential to be implemented on a flexible device and we propose caution when using action-specific tasks with bend gestures. 
We also compared two common handheld sizes. Results between the two sizes are similar leading us to believe the same gestures could be used for either device size. A follow up experiment testing the sizes independent would further confirm this finding. Finally, we were interested in observing the Simon Effect with gestures to see if users would associate the location of the gesture to the location of the stimuli. We found that larger displacement between stimuli follow the Simon Effect, hence, we recommend using gestures close to the stimulus.

Other interesting results include users matching opposing events to gestures paired by location. More specifically, participants used "push" and "pull" mental models. A majority of participants selected gestures that would "pull" the virtual object to move it. We also observed a variety of hand positions.

In conclusion, participants performed gestures in the location stimulus and paired gestures by location. We implement these findings as guidelines for our second experiment, where we evaluate the usability of the user-defined gestures and user experience with gaming. 


\section{Chapter: Experiment 2: Evaluating Usability and User Experience}

\section{$5.1 \quad$ Introduction}

We extended our exploration of gaming with flexible devices, by further examination of the usability of the user-defined gestures created from the first experiment. We extracted the main findings from the participatory experiment and implemented the user-defined gestures with six simple games: Pong, Bricks, PacMan, Tetris, Space Invaders and Fat Cats. We addressed the remaining unresolved events shooting, rotate and down-center by leveraging findings from prior research [42]. The Bendy prototype provided users with an immersive experience which instilled motivation and challenge through game play. In addition, it enabled us to implement the game controls and evaluate them in context, which was essential for our evaluation.

Our main research goals were to determine an overall preference for device size, evaluate the design of the game controls and observe behavioural patterns. We also sought to gain an in-depth understanding of users' gaming experience with flexible devices. We set our objectives to answer the following research questions:

R1: Was there evidence of a preference in size and why?

R2: How did the design of game controls perform in context?

R3: Did user behaviour relate to the Simon Effect?

R4: What are the observable bimanual interactions?

\subsection{Games}

This section describes each game and explains the justification behind the design of the controls. Each game was balanced to ease the game play for the participant. Where time was a factor the game pace was decreased (Tetris and Space Invaders). For the 
games with player lives this was increased to infinite to allow sufficient opportunity for the participant to experience the controls and game. The controls are the same for both device sizes, as we observed no meaningful differences during the first experiment.

\subsubsection{Pong}

Pong [2] is a classic arcade game, which consists of two paddles, one on each side of the screen. The goal of the game is to return the ball to the opponent's side. In the first experiment, the y-axis translation tasks showed a strong association between the location of the stimuli and the location of the gesture, in that participants were most likely to perform the gesture in the location of the stimuli. Furthermore, participants commonly paired opposing events by location. As a result, we placed the controls ipsilateral to the player's position. The participant was assigned the right paddle and used the top-rightcorner-down (G8) and bottom-right-corner-down (G6) to manipulate the position of the paddle. The researcher played using the left paddle against the participant.

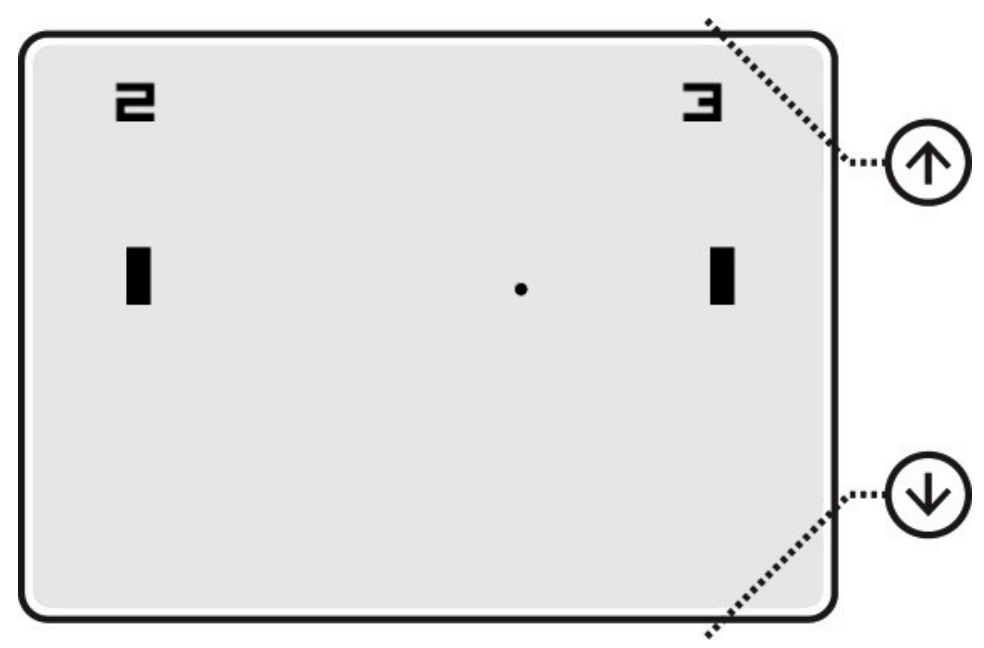

Figure 18 Game controls for Pong. 


\subsubsection{Bricks}

The game Bricks [3] is similar to Pong, however the paddle travels parallel to the $\mathrm{x}$-axis. The objective of the game is to deflect the ball using the paddle and eliminate the "bricks" above. The $\mathrm{x}$-axis translation task also indicated a strong association between the location of the stimuli and the location of the gesture. However, the results suggest that, regardless of stimuli location, there was a strong association to perform gestures in the center location using the left-side-down (G10) and right-side-down (G12) gestures. To manipulate the position of the paddle, participants will use the left and right sides of the device.

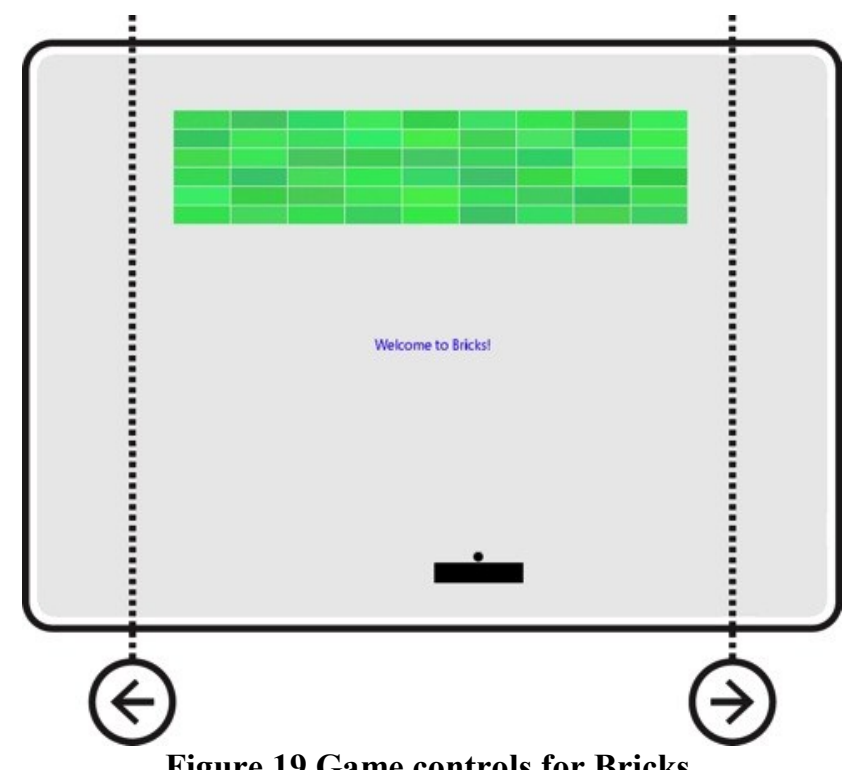

Figure 19 Game controls for Bricks.

\subsubsection{PacMan}

PacMan [29] is another popular arcade game where the player navigates a character, PacMan, through a maze and the objective is to eat all the pac-dots, while avoiding the enemies. In the XY translation tasks, participants did not reach a consensus for the up and down events. Our post conflict experiment did not resolve this conflict. 
While participants performed these events using the top and bottom sides of the device, we anticipated that these gestures would be difficult to execute during game play. Moreover, PacMan's position is continuously changing which poses an interesting design problem: how will users translate up or down when PacMan is on the left side or right side of the display? We leveraged our findings from the Simon Effect and placed the up and down controls on both sides of the device. We predicted that when PacMan was on the left side of the display participants would use the left side of the device and conversely, if PacMan were on the right participants would use the right side. To navigate through the maze, participants used left-side-down (G10) to go left, right-sidedown (G12) to go right, top- corners-down (G2 and G8) to go up and bottom-cornersdown (G4 and G6) to go down. This choice of gestures allowed us to remain consistent in our design decisions between games, as results showed that once participants developed a conceptual model they applied it consistently.

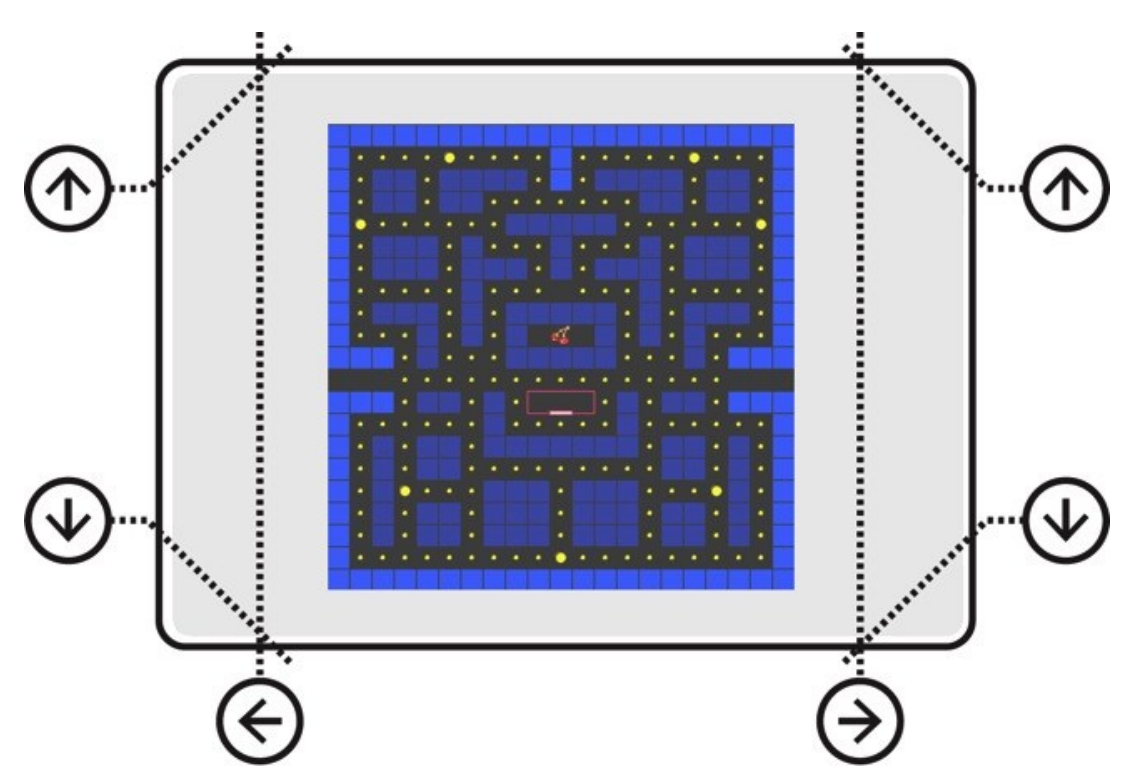

Figure 20 Game controls for PacMan. 


\subsubsection{Tetris}

Tetris [40] is a puzzle-based game where the objective is to manipulate the positioning of geometric shapes to form horizontal lines. The action-specific event rotate was unresolved after the post conflict experiment. However, participants reported associating the top-right-corner as the "trigger" button for action. Prior works recommend common functions be mapped to the top right corner and less frequent tasks to the bottom corners [42]. For Tetris, we assigned the rotate action to the top-corners-down (G2 and G8). Following our theory about the Simon Effect, we predicted that participants would use both sides of the device dependent on the object's location. We assigned the less frequent action "fast down" to the bottom-corners-down (G4 and G6). To manipulate the object left and right, participants will use left-side-down (G10) and right-side-down (G12), respectively.

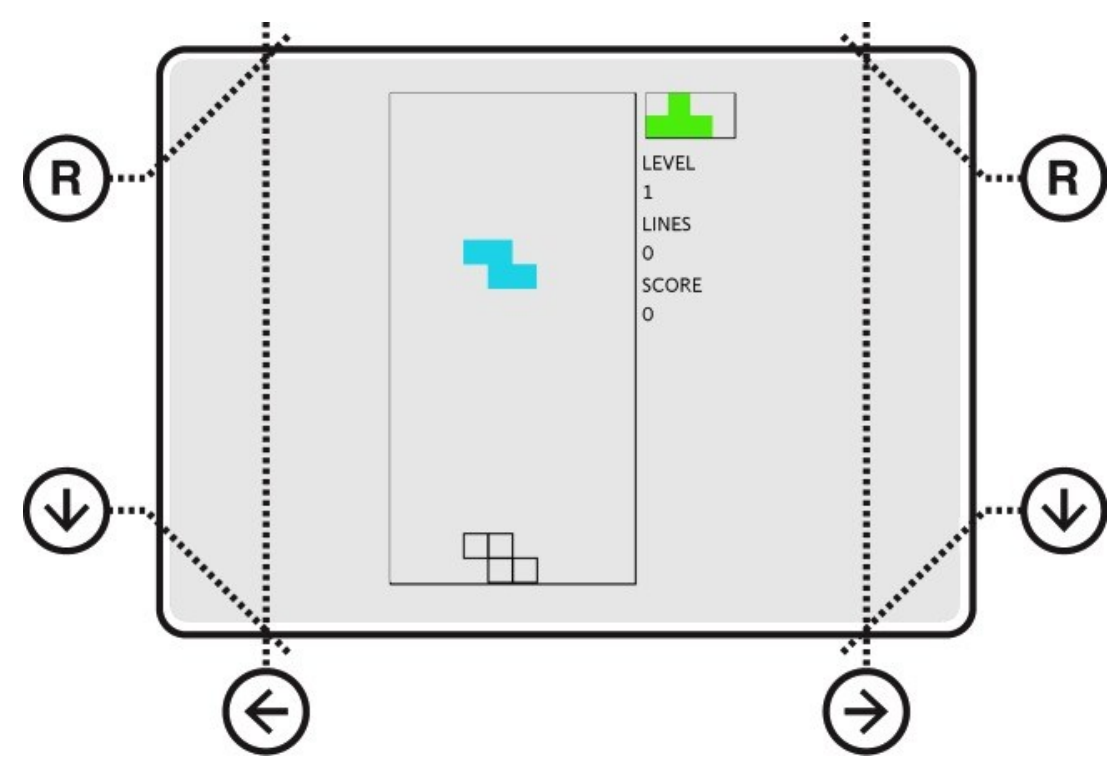

Figure 21 Game controls for Tetris 


\subsubsection{Space Invaders}

Space Invaders [38] is a two-dimensional shooter game. The player controls a space ship by moving it horizontally across the bottom of the screen, while shooting at the descending aliens above. We remained consistent in our design of the controls and mapped the shooting gesture to the top-corners-down (G2 and G8) and moving left and right using the sides of the device (G10 and G12).

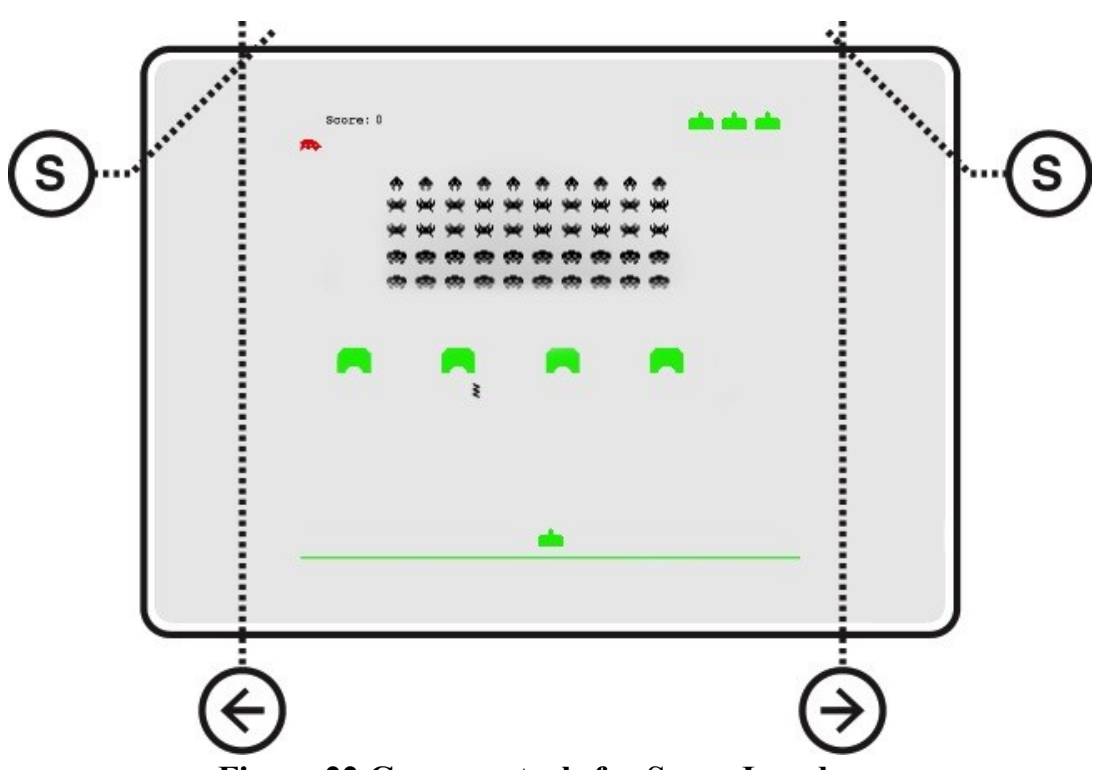

Figure 22 Game controls for Space Invaders.

\subsubsection{Fat Cats}

The design of Fats Cats was inspired by the popular game Angry Birds ${ }^{\mathrm{TM}}[32]$. The objective of the game is to fling the fat cat towards the scratching post aiming for one of three target levels. We applied our results from the spring task and assigned the left-side-down (G10) gesture to launch the Fat Cat. While the stimulus (cat) is located on the left side, we were unsure of how participants would perceive the target line. We predicted participants would perceive the cat being on the left side and use the left 
corners (G2 and G4) to manipulate the target line up and down. However, we also acknowledged the possibility that the directionality of the target line, as well as the direction of the flying cat, may influence participants' perceptions. To accommodate for these use scenarios, we placed the up and down controls on both sides of the devices.

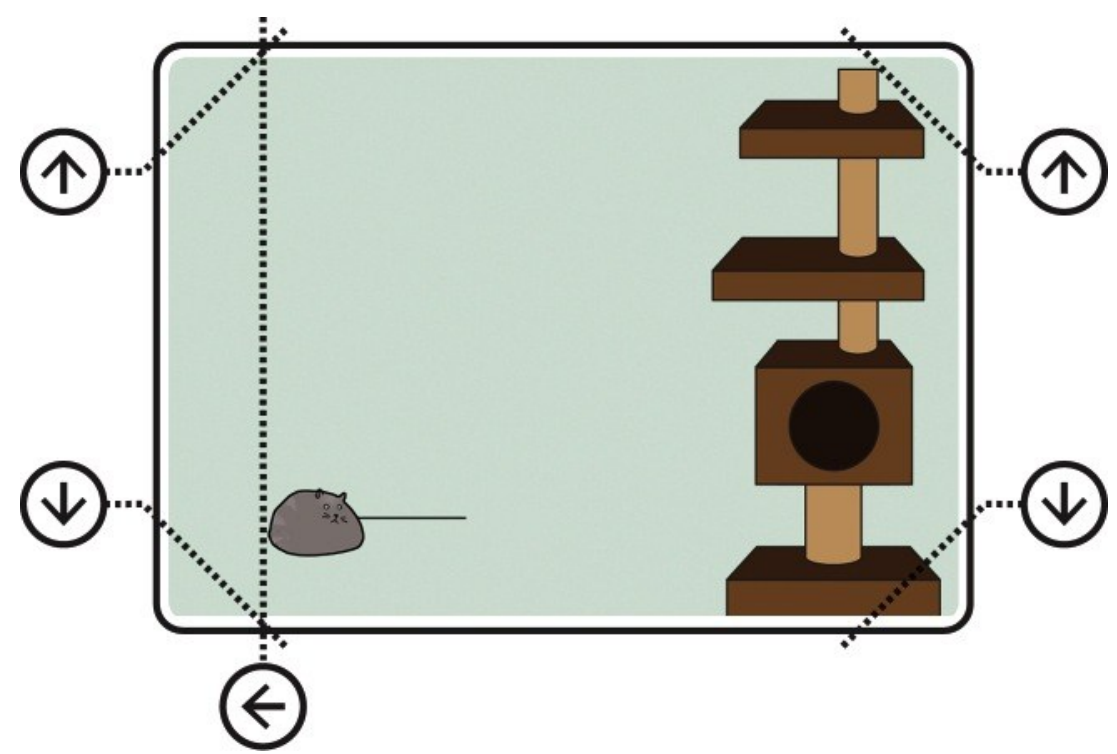

Figure 23 Game controls for Fat Cats.

\subsection{Participants}

12 participants (4 female) with no prior experience with flexible devices were recruited for the experiment. The average age was 24.25 years old. 10 participants were right handed, 1 left handed and 1 ambidextrous. Participants were given $\$ 10$ compensation. All participants owned a smartphone, 4 owned a tablet and 3 owned an eReader. Participants had no prior experience with bend gestures and did not participate in the first experiment. Participants reported to playing games occasionally (4.51/7) and had an average gaming experience. (4.08/7). 


\subsection{Methods}

Participants were given a brief introduction to flexible devices, bend gesture as an input for gaming and the prototype setup. The apparatus used can be found in Chapter 3 (Figure 11). Each session was approximately an hour in length. Prior to playing the games, participants were required to complete a tutorial, designed to teach them how to correctly perform the gestures. The tutorial provided visual feedback when the participant performed the gesture correctly. For example, when the top-right-corner was bent downwards an orange block on the top right quadrant would appear, if bent in the other direction the colour would change to blue. We devised the tutorial to familiarize participants with the gestures and improve accuracy in their performance. Once the participants felt comfortable using the prototype, we proceeded with the experiment. The order of the six games was randomized and the use of device size was counterbalanced. We designed a within-subjects experiment to evaluate each participant's perceptual differences between the two different sized prototypes. Participants played each game until they reported confidence in evaluating the controls.

To evaluate the usability of the controls for both sizes, we gathered quantitative data using two subjective metrics on a 7-point Likert scale: physical ease of use and mental intuitiveness. The questionnaire was administered after the participant played each game with both size devices. After the participant played all of the games with both sizes, they were asked to assess a "fun" metric that examined which games users found more fun with the use of bend gestures as an input. In order to determine an overall preference for size, users were asked to indicate their preferences after playing each game and to choose an overall size preference after playing all the games. We conducted a 
semi-structured interview during game play to solicit responses regarding the user's experience and asked the following open-ended questions:

a. How do you feel about the game controls?

b. How do you feel about the size of the device?

c. How do you feel about the difference in size?

Participants were also encouraged to think aloud while playing the games. The sessions were video recorded and detailed notes were taken. We gathered our qualitative data through detailed analysis of the videos and responses from the semi-structured interviews. In the next section, we report the outcomes of our analysis of the data collected.

\subsection{Results}

We gathered both quantitative and qualitative data to evaluate the usability and user experience of gaming with flexible devices.

\subsubsection{Quantitative}

To answer R1, if there was evidence of a preference in size, we tallied the results from the questionnaires. Participants preferred playing the games Pong (75\%), Bricks (67\%) and PacMan (58\%) using the small device. 58\% of participants preferred using the large device to play Tetris. For Space Invaders and Fat Cats, preference was divided equally amongst the sizes. When asked to indicate their overall preference, independent of a specific game, $75 \%$ of the participants preferred using the small device.

A Wilcoxon Signed-Rank test was run to determine if there were differences between the small and large size device for both physical and mental intuitiveness of the controls. There was no statistically significant difference between the sizes on both 
measures (physical ease of use: $\mathrm{z}=0.318, \mathrm{p}>.05$ mental intuitiveness: $\mathrm{z}=-1.00, \mathrm{p}>$ .05). Our qualitative data revealed further insight regarding the overall preference for the small device. While there was no statistical difference between small and large prototypes using those two metrics, qualitatively users offered several reasons for their preference of the small device and revealed some usability issues inherent to the larger device, which is discussed in section 5.2.2.

Our second research goal was to collect data according to how well the design of the controls were perceived by participants. We measured the physical ease of use and mental intuitiveness of the controls. On initial inspection of the averages of the scores we saw very little difference in the averages of the scores for the controls on both device sizes and across the six games as illustrated in Figure 24. To investigate further, we conducted a Friedman test to determine if there were differences between the games for each metric. There was a significant difference in the physical ease of use between the games on the small device $\chi^{2}(5)=12.049, \mathrm{p}<.05$. However, pairwise comparisons performed with a Bonferroni correction for multiple comparisons controlling for Type I errors were not significant. The results for physical ease of use on the large device $\left(\chi^{2}(5)\right.$ $=4.986, p>.05)$, and mental ease of use for both devices $\left(\operatorname{small} \chi^{2}(5)=7.883, p>.05\right.$, large $\left.\chi^{2}(5)=9.061, p>.05\right)$ between the games were not significant. In addition, the "fun" metric was also not significant $\left(\chi^{2}(5)=6.732, \mathrm{p}>.05\right)$. 


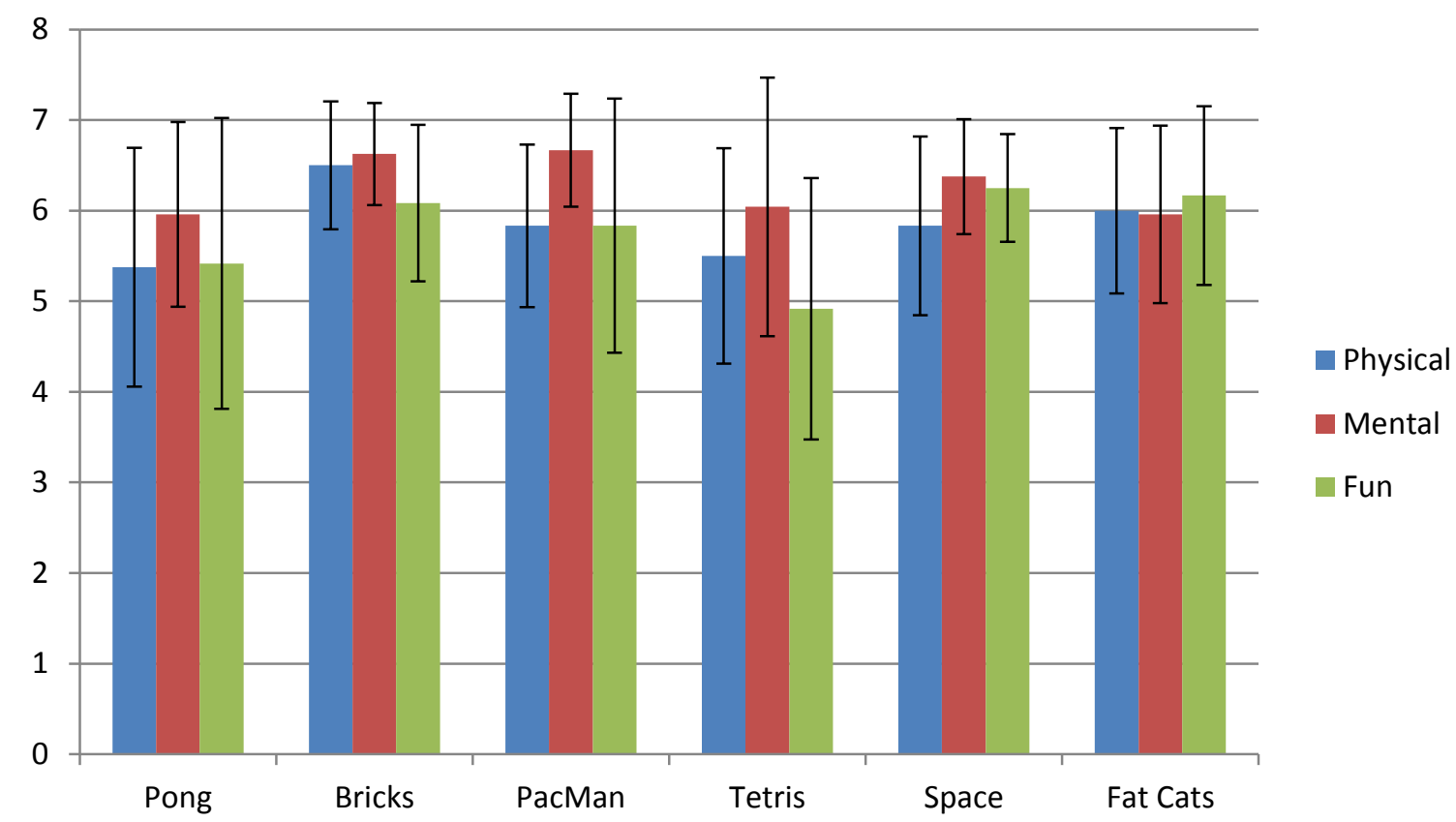

Figure 24 Averages of physical ease of use, mental intuitiveness and fun for all games.

\subsubsection{Qualitative data}

We gathered the notes taken from the videos and semi-structured interviews and analyzed the data using a descriptive coding procedure. The codes were categorized and used to help formulate our results.

\subsubsection{Preference for the Small Device}

Participants reported several reasons for their preferences of the small device. All participants commented on the distance between the controls and how it affected their performance. Most participants (10/12) found it easier to perform the gestures on the small device because they were closer together and required less hand movement. The smaller distance enabled participants to perform all the required gestures with minimal repositioning of the hands, which made it more comfortable to use and gave a greater sense of control. Participants' responses were "I like it [small device] better because of 
the smaller movements and I can brace the entire device." (P4) "The smaller one is better because the distance is closer, making them [controls] easier to access.” (P9)

On the large device, participants struggled to perform the gestures due to the increased distance between gestures. A couple of participants (2/12) favoured the larger distance between the controls because it felt more distinctive. "The bigger one [large device] was easier because they [the controls] were further apart and more distinct." (P1) All four female participants reported the large device being more difficult to use due to having smaller hands. A few of the male participants (3/8) indicated the larger device was slightly more comfortable because it fit their hand size better. Overall, the small device provided a better user experience because it was easier to use and increased game performance.

\subsubsection{Reducing the Need to Reposition Hands to Perform Gestures}

As previously stated, the designs of the controls were positively rated on both physical ease of use and mental intuitiveness. Contrary to the quantitative results of subjective metrics, all the participants commented on the repositioning of their hands to perform the gestures and preferred maintaining a close proximity to their hold position. Almost all the participants (10/12) struggled with the game Pong and suggested the gestures be collocated by direction instead of location to reduce repositioning of the hands. Two participants suggested positioning the device vertically and placing the controls on the bottom corner, which allowed them to hold the device and perform the gestures simultaneously.

While satisfied with the design of the controls, many of the participants $(9 / 12)$ commented that if the game pace were to increase they could envision the gestures being 
more difficult to execute. Some responses were "When you get further into Tetris and the game is faster, then it would get harder because you would have to move your hands more." (P1) "I think it would be more stressful if the game [PacMan] was faster." (P11) "It would be an injustice to expect someone to perform so many motions so fast." (P4)

Two participants found the bottom corner bends difficult. One participant reported a preference for the bottom bend by stating; "I like the fast down [in Tetris] on the bottom because I don't use it often and it's out of the way so I won't accidently hit it." (P12)

One-third of the participants suggested the combination of bend and touch input to decrease hand movement. "I like using bend to go side to side, but I would maybe tap the screen with my thumb to shoot [in Space Invaders].” (P5) For Tetris, one participant suggested using the accelerometer to rotate the Tetris pieces.

\subsubsection{Optimizing Game Performance}

A common theme that occurred during the experimental sessions were participants' attempts to optimize game performance using different strategies. The most common strategy (11/12) was experimenting with different hand positions and grips to optimize the use of all the gestures. P4 placed her fifth fingers on the bottom corners to perform gestures. P8 placed his index fingers on the top corners and thumbs on the sides of the device. Another strategy reported by a few participants (3/12) was wanting to use compound gestures as P10 suggested, "It would be great, if I could move and shoot [in Space Invaders] at the same time by bending the right side down and left side up." 


\subsubsection{Observable Patterns of the Simon Effect}

We analyzed the videos in detail and took note of whether participants exhibited behaviour governed by the Simon Effect. We designed the controls for PacMan, Tetris and Space Invaders by incorporating both sides of the device. For example if PacMan were on the left side of the display, we would expect that participants would use the controls on the left side, although they were available on both sides. All the participants used both sides of the device during PacMan, but only some (7/12) during Tetris and very few (3/12) during Space Invaders. During Space Invaders, half of the participants commented on the top right corner being similar to a game console controller. One participant submitted, "It's like using the buttons on my Xbox to shoot, which makes sense." (P7)

\subsubsection{Observable Bimanual Input Patterns}

All of the controls designed for the games required participants to perform asymmetrical actions using both hands. We observed two identifiable patterns in user behaviour. The first involved participants using one hand to hold the device and the other to perform the action. For Fat Cats, one-third of the participants preferred to use the controls on the left side and hold the device with their right hand. One participant suggested changing the controls for Bricks so that he could hold the device with his left hand and use only his right hand to manipulate the paddle.

The second pattern revealed participants assigning different types of actions to each hand. For Fat Cats, most of the participants $(8 / 12)$ used their left hand to launch the cat and the right hand to manipulate direction. We observed a unique behaviour from P7 as she used opposing sides to move and shoot while playing Space Invaders, whereas 
most participants $(8 / 12)$ only used the right corner. She commented, "I can play faster, if one hand is moving and the other is shooting."

\subsubsection{Potential for Gaming with Bend Gestures}

A majority of the participants self-reported and acknowledged (10/12) the potential of using bend gestures for gaming, specifically for the Bricks game. P10 and P11 compared the use of bend gestures as better than using their Blackberry joystick. Some participants $(5 / 12)$ commented bend gestures were better because it eliminated screen occlusion of the paddle. A majority of the participants $(8 / 12)$ found bend gestures to be a more tactile, interactive and interesting way to play a simple game compared to touch devices. One participant indicated it would be difficult to use touch to play Tetris and Space Invaders and that bend gestures are a better form of input.

\subsection{Discussion}

We observed and analyzed our data from both quantitative and qualitative perspectives in order to gain insight on the usability and user experience of gaming with flexible devices. In this section, we discuss the results and extract our main findings.

\subsubsection{Preference for Size}

While the quantitative results were not significant, participants provide detailed insight for the overall preference for the small size, which allows for greater ease of use, comfort and performance. However, a few participants indicated enjoying the larger display area of the large device. Some male participants, with larger hands enjoyed the fit. Research in the anthropometrics of human hands needs to be incorporated into the design of the flexible devices to accommodate for individual differences and improve user experiences. 


\subsubsection{User-Defined Gestures}

We implemented user-defined gestures for the game controls and found all of the results to be positive. However, evaluating the controls in this context allowed us to further extrapolate an important usability issue that did not arise during the participatory experiment. We found that having to reposition users' hands from the hold position to perform the gestures resulted in negative experiences. While the results from the first experiment strongly indicated that participants paired opposing events by location, the results of this experiment challenged this guideline, particularly for the y-axis translation in Pong. Pong received the most feedback for usability issues and participants most commonly suggested to collocate the gestures to a single location for ease of use. This also contradicts one of our findings from the participatory experiment: that most participants remained consistent in performing bend gestures once they conceptualized a mental model (push or pull). Participants' concern for better performance and usability outweighed our initial finding that opposing tasks were paired by location, which was driven by their mental model. Our evaluation proves to be beneficial in illuminating what is an important usability requirement, hand repositioning. An opportunity to further research hand repositioning during game play when using bend gestures would illuminate more information regarding this usability requirement.

\subsubsection{Using the Simon Effect for Translation Tasks}

Surprisingly, we found that participants did not use both sides of display as often as we had expected for Tetris and Space Invaders, compared to PacMan. This pattern was interesting to note due to the different genre of games compared. The Simon Effect 
appeared more prevalent in the translation type game (e.g. PacMan) and less in the action-specific games (e.g. Tetris and Space Invaders). This observation holds particularly true in the 2D shooter game where participants formed a strong association with the top-right-corner as the "trigger" button. In PacMan the gesture location was associated with the action performed, which may have made participants more inclined to utilize the full device.

\subsubsection{Bimanual Interaction Patterns}

Our observations of bimanual input led to behavioural patterns that follow Guiard's principles of bimanual interaction. Participants performed "coarse" actions with the non-preferred hand and more detailed and complex actions with the preferred hand. This was observed as two behavioural patterns during the Fat Cats game. The first observation involved participants holding the device with one hand and performing the actions with the other. In the second observation, we observed that participants assigned different types of actions to each hand. For instance, one hand was used to manipulate magnitude of the task and the other to perform an action. The later observation was more prominent during our second experiment because participants were able to perform better in the games if the division of tasks was assigned to both hands. While we observed this pattern, it was not within our research goals to examine in detail, however, we feel it is important to consider this interaction paradigm when designing games with flexible devices and therefore lays a foundation for future works. 


\subsection{Summary}

We evaluated Bendy using six games: Pong, Bricks, PacMan, Tetris, Space Invaders and Fat Cats. In conclusion, our qualitative provided insight for users overall preference for the small device as they found it was easier to use, more comfortable and achieved greater game performance. We found hand positioning as an important usability requirement that did not arise in the participatory experiment and triumphed over some of our initial design guidelines: pairing of events by location, which became a usability issue for Pong. The second contradiction was the guideline of consistency of user mental models, in which participants were willing to forego to increase game performance. Participants were unanimously concerned with having to reposition their hands from their holding positions to perform bend gestures. They disliked the increased hand movements and attributed this to decreased performance. In addition, we found observable user behaviour that confirmed our prediction of the Simon Effect and also identified bimanual interaction patterns. Overall, participants' responses were positive and acknowledged the potential for gaming with bend gestures. 


\section{Chapter: Conclusion}

In this thesis, we presented a comprehensive study into gaming with mobile flexible devices. We began our exploration by conducting a participatory experiment where users were asked to create bend gestures using the guessability methodology. Nine tasks were derived from simple games and grouped into translation, action-specific and physical manipulation. Our results provided a set of bend gestures for most of the tasks. We conducted a post-experiment, which resolved two of the five conflicting gestures that resulted from the first experiment. We found that the action-specific tasks scored the lowest in user agreement and the physical manipulations tasks ranked the highest. Additionally, we compared two common mobile device sizes and found similar bend gestures were created for each, leading us to believe the same gestures could be used for different sized mobile devices. However, an independent groups experiment would confirm this finding. Finally, we were interested in observing the Simon Effect with bend gestures and we found a strong association between the locations of the gesture to the location of the stimuli. Our preliminary investigation also illuminated interesting interaction paradigms such as users pairing opposing events to gestures by location, "push" and "pull" mental models, and experimenting with various hand positions. The

results served as guidelines for our implementation of the games in the second experiment.

Following the first experiment, we evaluated the user-defined gestures using six games: Pong, Bricks, PacMan, Tetris, Space Invaders and Fat Cats. We used the Bendy prototype, which is a flexible interactive prototype to evaluate participants' user experience and the usability of the game controls. In conclusion, there was an overall 
preference for the small device due to increased ease of use and performance. We found hand positioning as an important usability requirement to consider when designing flexible devices. Participants were unanimously concerned with having to reposition their hands from their holding positions to perform bend gestures. In addition, we confirm our prediction of the Simon Effect and identified novel interaction paradigms. We affirm our research goals that the use of bend gestures for gaming with mobile devices is a novel and viable input modality.

\subsection{Design Recommendations}

We propose the following eight design recommendations gathered from both experiments to help design future flexible devices and games:

1. Increase magnitude of bend gestures on larger devices.

On larger devices, the magnitude of the bend gestures should be greater to accommodate for individual differences in hand sizes and to minimize repositioning of the hands (Figure 25).

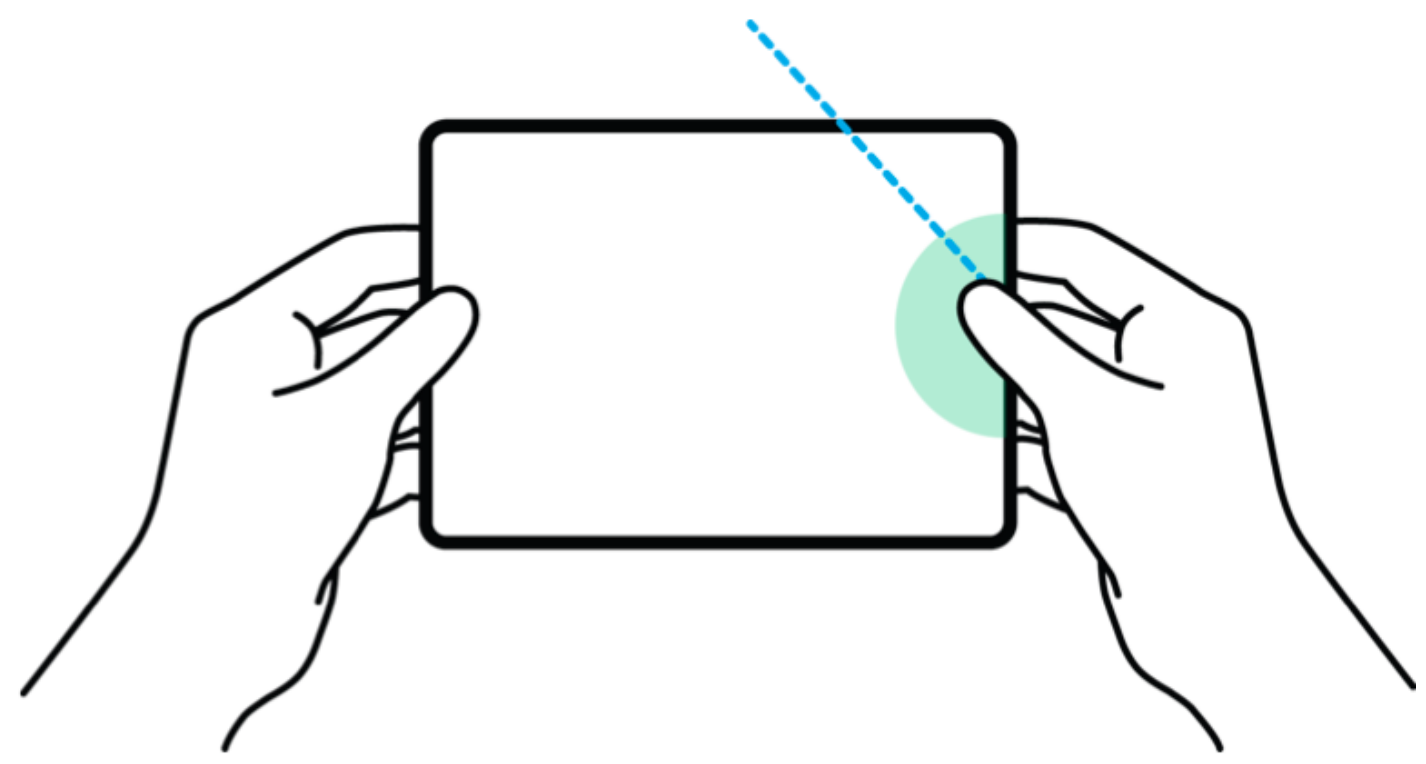

Figure 25 Increase magnitude of bend gesture for larger devices 
2. Keep bend gestures in close proximity to the hold position.

Due to a concordant consensus from participants, who reported negative experiences with frequent hand movements, we identified hand positioning as an important and usability requirement. We recommend when designing gestures for gaming the gestures be located in close proximity to the hold position minimizing movement. User feedback indicates that this will increase performance with game related tasks.

3. Use the left and right side bends for $x$-axis translation.

We found this had the highest user agreement and no related usability issues.

4. Use the top right corner to trigger action-specific events.

For games with action-specific events, participants created a strong association between the top-right-corner and the trigger on a game console controller. We recommend that this be used for repetitive and frequent actions.

5. Integrate both sides of the device for game control.

For devices with a fully flexible form factor, we recommend integrating both sides of the device and designing the controls to be redundant to accommodate for the Simon Effect.

6. Take advantage of real-world metaphor.

We recommend designers explore flexible interactions closely related to realworld actions because physical manipulation tasks ranked the highest in user consensus and the game Fat Cats was well received by participants. An example of leveraging a real-world metaphor is creating an archery-based game using the stretch physical deformation. 


\subsection{Limitations}

Bendy was based on an explorative process and as such our hardware, software, and methodologies were designed to fit our goals. We were limited by technological feasibility in available hardware. In lieu using a flexible display, we used projection. We made efforts to maintain the position of the display on the prototype. However, we were unable to reduce distortion during bend interactions. While the Bendy prototype provided users with an interactive experience, both hardware and software did not accommodate for individual differences in user performance, which was an unpredictable factor. Participants with larger hands struggled with performing the bend gestures to obtain accurate results. In addition, the bend gesture recognition software was limited to discrete functions, which restricted the use of finer metrics such as reaction time or performance errors. The quantitative metrics used in Experiment 2 were unable to capture significant values. We believe this could have been a result of the metrics being too board and unable to quantify the user experience. However, the qualitative data collected was meaningful and informed our research goals. Finally, our research goals were explorative and thusly we designed our methodology to fit within this scope, which also disallowed the use of finer metrics. Nevertheless, through our explorative study we have identified important usability requirements and interaction paradigms for future works.

\subsection{Future Works}

We envision exciting future works for Bendy. Our study explored an effective and innovative way of playing games on mobile devices using bend gestures. We would like to explore gaming interactions using other flexible concepts. Our research also led to interest findings in user behaviour such as the prevalence of the Simon Effect, division of 
bimanual interactions and various ergonomics consideration. What was revealing during research was the importance of the Simon Effect. It would be interesting to conduct a further study examining the relationship between the presence of Simon Effect and the physical distance between stimuli. We also identified two bimanual interaction patterns; future works include conducting an in-depth investigation on how users perform gaming tasks given bimanual input both symmetrical and asymmetrical. Finally, the games were selected for their simplicity in design. Further works would include designing games using real-world metaphors. For example squeezing a flexible device to illuminate a glow stick or flicking a device to toss paper into a trashcan. With improved deformation recognition techniques, we feel it would be important to conduct future in-depth works into these behaviours. Finally, we hope to further explore this work using a functional flexible display. 


\title{
Appendices
}

\section{Appendix A}

Questionnaires

\section{A.1 Post Experiment Questionnaire}

\author{
Pre-Test Questionnaire
}

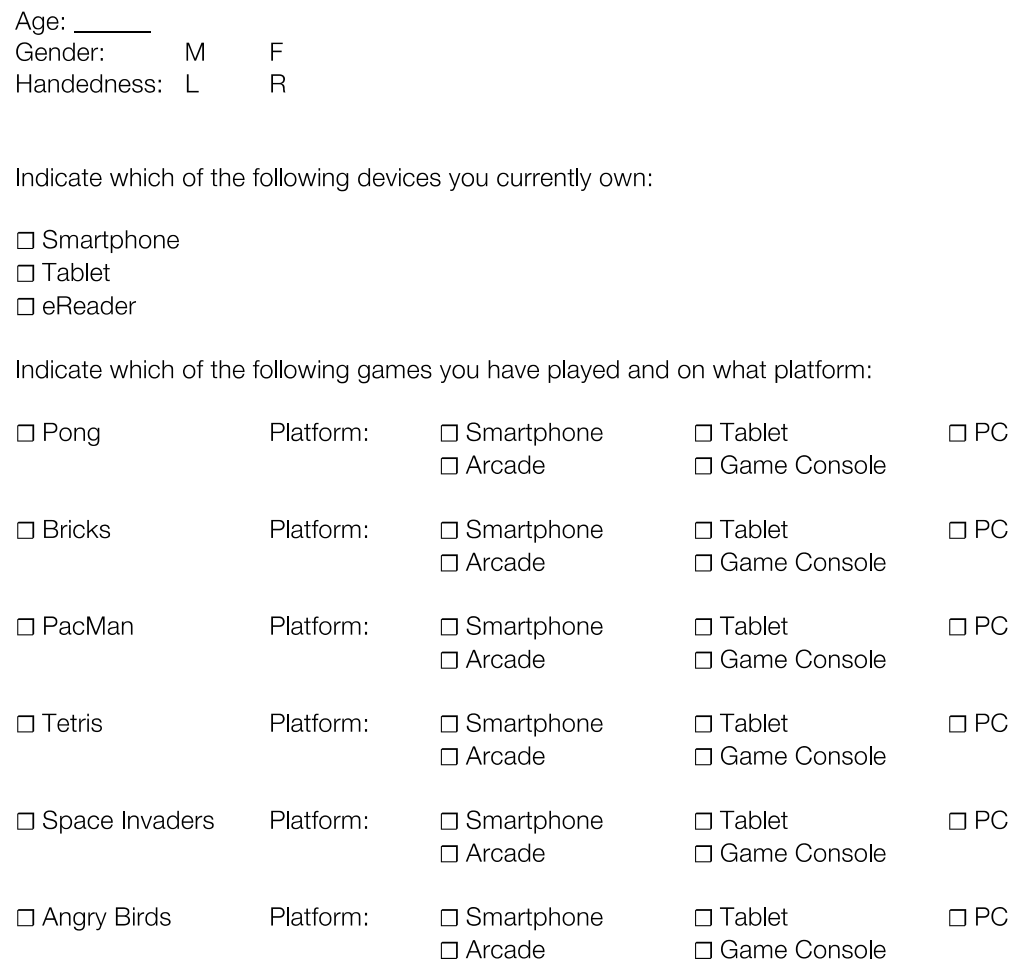

How often do you play mobile games?

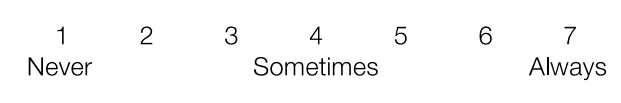

Indicate your level of expertise with mobile games.

$$
\begin{array}{ccccccc}
\begin{array}{c}
1 \\
\text { Beginner }
\end{array} & 2 & 3 & \begin{array}{c}
4 \\
\text { Average }
\end{array} & 5 & 6 & \begin{array}{c}
7 \\
\text { Expert }
\end{array}
\end{array}
$$




\section{A.2 Experiment 2 Questionnaire}

\begin{tabular}{|c|c|c|c|c|c|c|c|c|}
\hline \multirow[b]{2}{*}{ PONG } & & \multicolumn{2}{|c|}{$\begin{array}{l}\text { Strongly } \\
\text { Disagree }\end{array}$} & \multirow[b]{2}{*}{3} & \multirow[b]{2}{*}{4} & \multirow[b]{2}{*}{5} & \multicolumn{2}{|c|}{$\begin{array}{r}\text { Strongly } \\
\text { Agree }\end{array}$} \\
\hline & & 1 & 2 & & & & 6 & 7 \\
\hline \multirow[t]{2}{*}{ Small } & The controls were physically easy to perform. & o & o & o & o & o & o & o \\
\hline & The controls were mentally intuitive to use. & 0 & o & 0 & 0 & 0 & 0 & 0 \\
\hline \multirow[t]{3}{*}{ Large } & The controls were physically easy to perform. & o & o & o & o & 0 & o & o \\
\hline & The controls were mentally intuitive to use. & 0 & o & o & 0 & o & o & 0 \\
\hline & Overall, which size did you prefer? & & Small & & & & Large & \\
\hline \multicolumn{9}{|c|}{ BRICKS } \\
\hline \multirow[t]{2}{*}{ Small } & The controls were physically easy to perform. & o & o & o & o & o & o & o \\
\hline & The controls were mentally intuitive to use. & 0 & 0 & o & 0 & 0 & 0 & 0 \\
\hline \multirow[t]{3}{*}{ Large } & The controls were physically easy to perform. & 0 & 0 & o & 0 & 0 & o & 0 \\
\hline & The controls were mentally intuitive to use. & o & 0 & o & o & o & o & o \\
\hline & Overall, which size did you prefer? & & Small & & & & Large & \\
\hline \multicolumn{9}{|c|}{ PACMAN } \\
\hline \multirow[t]{2}{*}{ Small } & The controls were physically easy to perform. & 0 & 0 & o & 0 & 0 & o & 0 \\
\hline & The controls were mentally intuitive to use. & o & o & o & o & 0 & o & 0 \\
\hline \multirow[t]{3}{*}{ Large } & The controls were physically easy to perform. & o & 0 & o & o & o & o & o \\
\hline & The controls were mentally intuitive to use. & 0 & 0 & o & 0 & 0 & o & 0 \\
\hline & Overall, which size did you prefer? & & Small & & & & Large & \\
\hline \multicolumn{9}{|c|}{ TETRIS } \\
\hline \multirow[t]{2}{*}{ Small } & The controls were physically easy to perform. & o & 0 & o & o & o & o & o \\
\hline & The controls were mentally intuitive to use. & 0 & 0 & o & 0 & 0 & o & 0 \\
\hline \multirow[t]{3}{*}{ Large } & The controls were physically easy to perform. & 0 & 0 & o & 0 & 0 & 0 & 0 \\
\hline & The controls were mentally intuitive to use. & 0 & 0 & 0 & 0 & 0 & 0 & 0 \\
\hline & Overall, which size did you prefer? & & Small & & & & Large & \\
\hline \multicolumn{9}{|c|}{ SPACE INVADERS } \\
\hline \multirow[t]{2}{*}{ Small } & The controls were physically easy to perform. & 0 & 0 & 0 & 0 & 0 & 0 & 0 \\
\hline & The controls were mentally intuitive to use. & 0 & $\circ$ & 0 & 0 & o & 0 & 0 \\
\hline \multirow[t]{3}{*}{ Large } & The controls were physically easy to perform. & 0 & 0 & o & 0 & 0 & o & 0 \\
\hline & The controls were mentally intuitive to use. & o & 0 & 0 & 0 & 0 & 0 & 0 \\
\hline & Overall, which size did you prefer? & & Small & & & & Large & \\
\hline \multicolumn{9}{|c|}{ FAT CAT } \\
\hline \multirow[t]{2}{*}{ Small } & The controls were physically easy to perform. & 0 & o & o & o & 0 & o & 0 \\
\hline & The controls were mentally intuitive to use. & 0 & 0 & 0 & 0 & 0 & 0 & 0 \\
\hline \multirow[t]{3}{*}{ Large } & The controls were physically easy to perform. & 0 & 0 & o & 0 & 0 & o & 0 \\
\hline & The controls were mentally intuitive to use. & 0 & 0 & 0 & 0 & 0 & 0 & 0 \\
\hline & Overall, which size did you prefer? & & Small & & & & Large & \\
\hline
\end{tabular}




\begin{tabular}{|c|c|c|c|c|c|c|c|c|}
\hline & & \multicolumn{2}{|c|}{$\begin{array}{l}\text { Strongly } \\
\text { Disagree }\end{array}$} & \multirow[b]{2}{*}{3} & \multirow[b]{2}{*}{4} & \multirow[b]{2}{*}{5} & \multicolumn{2}{|c|}{$\begin{array}{r}\text { Strongly } \\
\text { Agree }\end{array}$} \\
\hline \multicolumn{2}{|c|}{ For each game evaluate the following: } & 1 & 2 & & & & 6 & 7 \\
\hline Pong & Bend gestures were fun. & 0 & 0 & 0 & 0 & 0 & 0 & 0 \\
\hline Bricks & Bend gestures were fun. & 0 & 0 & 0 & 0 & 0 & 0 & 0 \\
\hline Pacman & Bend gestures were fun. & 0 & 0 & 0 & 0 & 0 & 0 & 0 \\
\hline Tetris & Bend gestures were fun. & 0 & 0 & 0 & 0 & 0 & 0 & 0 \\
\hline Space Invaders & Bend gestures were fun. & 0 & 0 & 0 & 0 & 0 & 0 & 0 \\
\hline Fat Cat & Bend gestures were fun. & 0 & 0 & 0 & 0 & 0 & 0 & 0 \\
\hline
\end{tabular}

$\begin{array}{lll}\text { Overall, which size did you prefer? } & \text { Small Large }\end{array}$

Rank your preference for the use of bend gesture as an input. 1 being the most preferred.

Pong

Brick

Pacman

Tetris

Space Invaders

Fat Cat 


\section{References}

1. Arduino. Arduino Uno. http://arduino.cc/en/Main/arduinoBoardUno.

2. Atari Arcade. Pong. http://atari.com/arcade\#!/arcade/pong/play.

3. Atari Arcade. Breakout. http://atari.com/arcade\#!/arcade/superbreakout/play.

4. Balakrishnan, R. and Fitzmaurice, G. Exploring interactive curve and surface manipulation using a bend and twist sensitive input strip. Proc. Interactive $3 D$ graphics, ACM (1999), 111-119.

5. Browne, K. and Anand, C. An empirical evaluation of user interfaces for a mobile video game. Entertainment Computing 3, 1 (2012), 1-10.

6. Casual Game Association. Casual Games Market Report 2007. 2007.

7. Chehimi, F. and Coulton, P. Motion controlled mobile 3D multiplayer gaming. Proc. ACE, ACM (2008), 267-270.

8. Daniel, B., Wenig, D., and Malaka, R. Mobile Gaming with Indirect Sensor Control. Entertainment Computing, Springer Berlin Heidelberg (2012), 441-444.

9. Fishkin, K.P., Gujar, A., Harrison, B.L., Moran, T.P., and Want, R. Embodied user interfaces for really direct manipulation. Communications of the ACM 43, 9 (2000), 74-80.

10. Fitts, P.M. The information capacity of the human motor system in controlling the amplitude of movement. Journal of Experimental Psychology 47, (1954), 381391.

11. Flexpoint. http://www.flexpoint.com. .

12. Gallant, D., Seniuk, A., and Vertegaal, R. Towards more paper-like input: flexible input devices for foldable interaction styles. Proc. UIST, ACM (2008), 283-286.

13. Gilbertson, P., Coulton, P., Chehimi, F., and Vajk, T. Using "tilt" as an interface to control "no-button" 3-D mobile games. Computers in Entertainment 6, 3 (2008), $1-13$.

14. Guiard, Y. Asymmetric division of labor in human skilled bimanual action: the kinematic chain as a model. Journal of motor behavior 19, 4 (1987), 486-517. 
15. Herkenrath, G., Karrer, T., and Borchers, J. Twend: twisting and bending as new interaction gesture in mobile devices. CHI'08 Extended Abstracts, ACM (2008), $3819-3824$.

16. Hick, W. On the rate of gain of information. Journal of Experimental Psychology 4, (1952), 11-36.

17. Holman, D. and Vertegaal, R. Organic User Interfaces : Design Computer in Any Way, Shape, or Form. Communications of the ACM 51, 6 (2008).

18. Hyman, R. Stimulus information as a determinant of reaction time. Journal of Experimental Psychology 45, (1953), 188-196.

19. Ishii, $\mathrm{H}$. The tangible user interface and its evolution. Communications of the $A C M$ $51,6(2008), 32$.

20. Jacob, R.J.K., Girouard, A., Hirshfield, L.M., et al. Reality-based interaction: a framework for post-WIMP interfaces. Proc. CHI, ACM (2008), 201-210.

21. Kaltenbrunner, M. and Bencina, R. reacTIVision: a computer-vision framework for table-based tangible interaction. Proc. TEI, ACM (2007), 15-17.

22. Kildal, J., Paasovaara, S., and Aaltonen, V. Kinetic Device: Designing Interactions with a Deformable Mobile Interface. Proc. CHI EA, (2012), 18711876.

23. Kildal, J. and Wilson, G. Feeling it: the roles of stiffness, deformation range and feedback in the control of deformable UI. Proc. ICMI, ACM (2012), 393-400.

24. Lahey, B. and Girouard, A. PaperPhone: understanding the use of bend gestures in mobile devices with flexible electronic paper displays. Proc. CHI, 2011, 13031312. http://dl.acm.org/citation.cfm?id=1979136.

25. Lee, S., Lim, Y., and Lee, K.-P. Exploring the effects of size on deformable user interfaces. Proc. MobileHCI, (2012), 89.

26. Lee, S., Maeng, S., Kim, D., Lee, K., and Lee, W. FlexRemote: Exploring the Effectiveness of Deformable User Interface as an Input Device for TV. HCI International 2011-Posters' Extended Abstracts, Springer Berlin Heidelberg (2011), 62-65.

27. Lee, S.-S., Kim, S., Jin, B., et al. How users manipulate deformable displays as input devices. Proc. CHI, ACM (2010), 1647. 
28. McNaughton, M. Mobile Gaming To Rise 26\%, Social Gaming Up 10\% in 2012. 2012. http://therealtimereport.com/2012/06/12/mobile-gaming-to-rise-26-socialgaming-up-10-in-2012/.

29. Namco Bandai Games. Pacman. http://pacman.com/.

30. Optoma. Optoma PK301+. http://optomausa.com/products/detail/PK301plus.

31. Proctor, R.W. and Reeve, T.G. Stimulus-response compatibility: An intergrated perspective. Elsevier, 1989.

32. Rovio Entertainment. Angry Birds. http://www.angrybirds.com/.

33. Samsung. Samsung Highlights Innovations in Mobile Experiences Driven by Components, in CES Keynote. http://www.samsung.com/global/business/semiconductor/news-events/pressreleases/detail?newsId=12521.

34. Schwesig, C., Poupyrev, I., and Mori, E. Gummi: a bendable computer. Proc. CHI, ACM (2004), 263-270.

35. Siek, K.A., Rogers, Y., and Connelly, K.H. Fat finger worries: how older and younger users physically interact with PDAs. Human Computer Interaction INTERACT, Springer Berlin Heidelberg (2005), 267-280.

36. Simon, J.R. Reactions toward the source of stimulation. J Exp Psychology 8, 1 (1969), 174-176.

37. Steimle, J., Jordt, A., and Maes, P. Flexpad: highly flexible bending interactions for projected handheld displays. Proc. CHICHI, ACM (2013).

38. Taito Corporation. Space Invaders. http://spaceinvaders.net/.

39. Tajika, T., Yonezawa, T., and Mitsunaga, N. Intuitive page-turning interface of ebooks on flexible e-paper based on user studies. Proc. MM, ACM (2008), 793.

40. Tetris. Tetris. http://www.tetris.com/.

41. The Nielsen Company. The State Of Mobile Apps. 2011.

42. Warren, K., Lo, J., Vadgama, V., and Girouard, A. Bending the rules: bend gesture classification for flexible displays. Proc. CHI, (2013).

43. Watanabe, J., Mochizuki, A., and Horry, Y. Bookisheet: bendable device for browsing content using the metaphor of leafing through the pages. Proc. UbiComp, (2008), 360-369. 
44. Wightman, D., Ginn, T., and Vertegaal, R. BendFlip: Examining Input Techniques for Electronic Book Readers with Flexible Form Factors. Proc. INTERACT, (2011), 117-133.

45. Wobbrock, J.O., Aung, H.H., Rothrock, B., and Myers, B. a. Maximizing the guessability of symbolic input. Extended abstracts - CHI, ACM (2005), 1869.

46. Wobbrock, J.O., Morris, M.R., and Wilson, A.D. User-defined gestures for surface computing. Proc. CHI, ACM (2009), 1083.

47. Ye, Z. and Khalid, H. Cobra: flexible displays for mobilegaming scenarios. Extended abstracts - CHI, ACM (2010), 4363-4367.

48. Zaman, L., Natapov, D., and Teather, R.J. Touchscreens vs. traditional controllers in handheld gaming. Proc. Futureplay, ACM (2010), 183.

49. SNES.jpg. http://upload.wikimedia.org/wikipedia/commons/c/cf/SNES-Controllerin-Hand.jpg. 